JOURNAL OF THE AMERICAN MATHEMATICAL SOCIETY

Volume 10, Number 1, January 1997, Pages 139-167

S 0894-0347(97)00222-1

\title{
STRUCTURE OF A HECKE ALGEBRA QUOTIENT
}

\author{
C. KENNETH FAN \\ Dedicated to my teacher, George Lusztig, on his fiftieth birthday
}

\section{INTRODUCTION}

Let $W$ be a Coxeter group with Coxeter graph $\Gamma$. Let $\Gamma_{g}$ be the set of simple generators, which are parametrized by the nodes of $\Gamma$.

Our primary interest in this paper is to understand the case where $\Gamma$ is of type $E$. Therefore, we shall assume that $\Gamma$ is of type $A, D$, or $E$, where by $E$, we mean the infinite series $E_{n}$ which begins $E_{5}=D_{5}, E_{6}, E_{7}, E_{8}, E_{9}=\hat{E}_{8}$, etc.

Every $w \in W$ may be written as a product $s_{1} s_{2} s_{3} \cdots s_{n}$ of generators in $\Gamma_{g}$. If $n$ is minimal, we call this product "reduced" and define $l(w)=n$. More generally, if $w=w_{1} w_{2} w_{3} \cdots w_{n}$ satisfies $l(w)=\sum_{i} l\left(w_{i}\right)$, then we call this product "reduced" as well.

Let $\mathcal{H}$ be the Iwahori-Hecke algebra associated to $W$. This is an algebra over $\mathbb{Q}\left(q^{1 / 2}\right)$ (where $q^{1 / 2}$ is an indeterminate) with generators $T_{s}$ for each $s \in \Gamma_{g}$ satisfying the relations $T_{s}^{2}=(q-1) T_{s}+q, T_{s} T_{t}=T_{t} T_{s}$ if $s t=t s$, and $T_{s} T_{t} T_{s}=T_{t} T_{s} T_{t}$ if $s t s=t s t$, where $s, t \in \Gamma_{g}$. This algebra has a basis $T_{w}, w \in W$, where we have $T_{w}=T_{s_{1}} \cdots T_{s_{n}}$ whenever $s_{1} \cdots s_{n}$ is a reduced expression for $w$.

Let $\mathcal{I}$ be the two-sided ideal generated by the elements

$$
T_{s t s}+T_{s t}+T_{t s}+T_{s}+T_{t}+1
$$

where we have one such expression for each pair of non-commuting generators $s$, $t \in \Gamma_{g}$. (In fact, it suffices, since $\Gamma$ is connected, to take one such expression for one pair of non-commuting generators.)

Define $\overline{\mathcal{H}}=\mathcal{H} / \mathcal{I}$.

When $W$ is a Coxeter group of type $A$, this is known as the Temperley-Lieb algebra, which has been studied by Jones [8], who attributes the notion to Temperley and Lieb [16]. For more details on this history, see [9, p. 104]. Apparently, there is a considerable body of literature devoted to this algebra. For instance, Westbury [17] describes a realization of this algebra related to braids. Also, Kauffmann and Saleur [10] give a formulation (which has a direct generalization to all types) of the four color theorem in terms of multiplication in the Temperley-Lieb algebra. For more general Coxeter groups, this algebra has been defined and studied in [2], [3], and [7]. In particular, in Graham's thesis [7], Graham proves that these algebras are "cellular". We are not certain how the results of this paper are related to those

Received by the editors May 14, 1996.

1991 Mathematics Subject Classification. Primary 16G30, 05E99; Secondary 16D70, 20F55.

Key words and phrases. Iwahori-Hecke algebra, Temperley-Lieb algebra, Coxeter group, cell theory, semi-simple algebra.

Supported in part by a National Science Foundation postdoctoral fellowship. 
of [7], although the referee has pointed out that Graham's results on cellularity are closely related to the results of this paper concerning left, right, and two-sided cells, and especially with the fact that each left and right cell in the same two-sided cell intersect in a single element (see proof of theorem 6.1.2).

In this paper, the main result is the semi-simplicity of $\overline{\mathcal{H}}$ in type $E$ (section 5 ) and an understanding of the dimensions of the irreducible representations (section 6). This amounts to a classification of the finite, generically simple representations of $\mathcal{H}$ on which $\mathcal{I}$ acts trivially. We shall prove semi-simplicity by proving non-degeneracy of a certain bilinear form.

In the appendix, we gather together some combinatorial facts about reduced expressions in type $E$ which we need in section 6 .

It was shown in [7] that the algebra $\overline{\mathcal{H}}$ can be generalized to all Coxeter groups and that the algebra is finite dimensional when $\Gamma$ is from one of the seven infinite series of graphs $A_{n}, B_{n}, D_{n}, E_{n}, F_{n}, H_{n}$, and $I_{2}(m)$ (where $I_{2}(m)$ denotes the series of dihedral groups). In section 7 , we shall discuss the cases where $\Gamma$ is of type $B, F$, or $H$. We believe that $\overline{\mathcal{H}}$ is semi-simple whenever $\overline{\mathcal{H}}$ is finite-dimensional and can prove this in all cases except type $H$.

We thank Richard Green, Benedict Gross, Patricia Hersh, David Kazhdan, Amie Wilkinson, and the referee for beneficial conversations and comments. We also thank George Lusztig, without whom this paper would not exist.

\section{Preliminaries}

2.1. The subset $W_{c}$ of $W$. Let $w \in W$. Define the support of $w$ to be the set of generators which appear in some (or any) reduced word for $w$. We shall also denote this by $\operatorname{supp}(w)$. Define $\mathcal{L}(w)$ to be the set of $s \in \Gamma_{g}$ such that $l(s w)<l(w)$. Similarly, define $\mathcal{R}(w)$ to be the set of $s \in \Gamma_{g}$ such that $l(w s)<l(w)$.

Denote by $W_{c}$ those elements of $W$ whose reduced expressions avoid substrings of the form sts where $s$ and $t$ are non-commuting generators in $\Gamma_{g}$. These elements have been studied in [2], [3], [4], [6], [14], [15].

Throughout this paper we shall use many properties of reduced expressions for elements of $W$ and, especially, for elements of $W_{c}$. We summarize the main properties here.

\section{Properties}

R1. (Iwahori-Tits) Every reduced word for $w$ can be obtained from any other by applying a sequence of braid relations.

R2. If $w \in W_{c}$, every reduced expression for $w$ can be obtained from any other by applying a sequence of commutation relations.

R3. Let $s_{i_{1}} s_{i_{2}} s_{i_{3}} \cdots s_{i_{n}}$ be a sequence of generators in the set $\Gamma_{g}$. Then $w=$ $s_{i_{1}} s_{i_{2}} s_{i_{3}} \cdots s_{i_{n}}$ is reduced and in $W_{c}$ if and only if between any two occurrences of a generator $s$ in the sequence, there occurs at least two instances of generators which do not commute with $s$.

R4. Suppose $w \in W_{c}, t \in \Gamma_{g}$ and $w t \notin W_{c}$. Then, there exists a unique $s \in S$ such that every reduced expression of $w$ may be parsed uniquely as follows: $w=$ $w_{1} t w_{2} s w_{3}$ reduced, $(s t)^{3}=1$, and $t$ commutes with every $u \in \operatorname{supp}\left(w_{2}\right) \cup \operatorname{supp}\left(w_{3}\right)$.

$\mathrm{R} 4^{\prime}$. Suppose $w \in W_{c}, t \in \Gamma_{g}$ and $t w \notin W_{c}$. Then, there exists a unique $s \in S$ such that every reduced expression of $w$ may be parsed uniquely as follows: $w=$ $w_{1} s w_{2} t w_{3}$ reduced, $(s t)^{3}=1$, and $t$ commutes with every $u \in \operatorname{supp}\left(w_{1}\right) \cup \operatorname{supp}\left(w_{2}\right)$. 
R5. Let $w \in W_{c}, t \in S$. Assume $w t \notin W_{c}$. Then we may write $w=w_{1} t s w_{2}$ reduced, $t$ commutes with all $u \in \operatorname{supp}\left(w_{2}\right)$, and $(s t)^{3}=1$. Furthermore, if $w=$ $w_{1}^{\prime} t s^{\prime} w_{2}^{\prime}$ is another such expression, then $s=s^{\prime}$.

$\mathrm{R} 5{ }^{\prime}$. Let $w \in W_{c}, t \in S$. Assume $t w \notin W_{c}$. Then we may write $w=w_{1} s t w_{2}$ reduced, $t$ commutes with all $u \in \operatorname{supp}\left(w_{1}\right)$, and $(s t)^{3}=1$. Furthermore, if $w=$ $w_{1}^{\prime} s^{\prime} t w_{2}^{\prime}$ is another such expression, then $s=s^{\prime}$.

R6. Let $w \in W_{c}$. Then $\mathcal{L}(w)$ and $\mathcal{R}(w)$ each consist of mutually commuting nodes.

For property R1, see [1]. Properties R2 and R3 are corollaries of property R1. A proof of property $\mathrm{R} 4^{\prime}$ may be found in [2] or [3]. Property $\mathrm{R} 5^{\prime}$ follows from property R4'. Properties R4 and R5 are dual to R4 $4^{\prime}$ and R5'. Property R6 is proven in [2].

Let $w \in W_{c}$. Let $w=s_{i_{1}} s_{i_{2}} s_{i_{3}} \cdots s_{i_{n}}$ be a reduced expression for $w$. The set consisting of labels for each of the terms in this sequence (using different labels for multiple occurrences of the same generator) can be given the structure of a partially ordered set in which labels corresponding to the same generator form a totally ordered subset, and incomparable elements commute. This fact can be deduced from [2, p. 14]. The partially ordered set is known as the "heap" of $w$. Stembridge [14] has unearthed many properties of the heap. Many of our results say something about heaps, but we leave this translation to the reader.

2.2. The monomial basis of $\overline{\mathcal{H}}$. Let $t_{w}$ be the projection of $T_{w}$ to $\overline{\mathcal{H}}$.

Let $b_{s}=q^{-1 / 2}\left(t_{s}+1\right) \in \overline{\mathcal{H}}$. It was shown in [2] that $\overline{\mathcal{H}}$ is isomorphic to the $\mathbb{Q}\left(q^{1 / 2}\right)$-algebra generated by the $b_{s}, s \in \Gamma_{g}$, with the following relations: $b_{s}^{2}=q_{2} b_{s}$, $b_{s} b_{t}=b_{t} b_{s}$ if $s t=t s$, and $b_{s} b_{t} b_{s}=b_{s}$ if $s t s=t s t$, where $q_{2}=q^{1 / 2}+q^{-1 / 2}$. For $w \in W_{c}$, it makes sense to define $b_{w}=b_{s_{1}} \cdots b_{s_{n}}$ where $w=s_{1} \cdots s_{n}$ is a reduced expression for $w$. The elements $b_{w}, w \in W_{c}$, constitute a basis of $\overline{\mathcal{H}}$.

Note that the structure constants with respect to the basis $b_{w}, w \in W_{c}$, belong to $\mathbb{Z}\left[q^{1 / 2}, q^{-1 / 2}\right]$. Indeed, for any $w, w^{\prime} \in W_{c}$, there exist a nonnegative integer $m$ and $w^{\prime \prime} \in W_{c}$ such that $b_{w} b_{w^{\prime}}=q_{2}{ }^{m} b_{w^{\prime \prime}}$. By "positivity of structure constants", we are referring to the fact that $m$ is a nonnegative integer. Later, we shall refine our understanding of the structure constants (see proposition 5.4.1).

2.3. More notation. Let $P$ denote the set of subsets of $\Gamma$ which consist of nonadjacent nodes. We allow $P$ to include the empty set, $\emptyset$. For any $S \in P$, let $i(S)$ be the product of the simple generators corresponding to the nodes in $S$ (with $i(\emptyset)=1)$. Note that the order of the product is immaterial since the nodes in $S$ are non-adjacent.

Let $S, T \in P$. We say that $S$ and $T$ are neighbors if and only if $1+\# S \cap T=$ $\# S=\# T$, and the two nodes in $(S \cup T) \backslash(S \cap T)$ are joined in $\Gamma$. Define an equivalence relation $\sim$ on $P$ by taking the transitive closure of the relation $S \sim T$ if $S$ and $T$ are neighbors. Let $\bar{P}$ denote the set $P / \sim$.

For any $w \in W_{c}$, we define a nonnegative integer $a(w)$ as follows.

Definition 2.3.1. Let $w \in W_{c}$. Define

$$
a(w)=\max _{S \in P}\{\# S \mid i(S) \text { is a substring of some reduced expression for } w\} .
$$

When $W$ is finite, it can be shown that $a(w)$ is the restriction to $W_{c}$ of the $a$-function defined by Lusztig in [12], and the referee has suggested that this fact 
may be true in general. In terms of the heap of $w, a(w)$ is the size of a maximal anti-chain.

Observe that if $l(s w)>l(w)$, then $a(s w) \geq a(w)$ since any substring of a reduced expression for $w$ would be a substring for a reduced expression for $s w$. Also note that $a(i(S))=\# S$.

\section{InVOLUTIONS IN $W_{c}$}

3.1. Canonical form for involutions. Eventually, we shall show that the number of involutions in $W_{c}$ is equal to the sum of the dimensions of the irreducible representations (see corollary 6.1.4). For now, we give a canonical form for involutions in $W_{c}$.

Theorem 3.1.1. Let $w \in W_{c}$ satisfy $w^{2}=1$. There exist a unique $S \in P$ and a unique $x \in W_{c}$ such that $w=x i(S) x^{-1}$ reduced.

Proof. We first show existence.

We construct a finite sequence of triples $\left(w_{n}, x_{n}, S_{n}\right)$ for $0 \leq n \leq N$, such that $w_{n}, x_{n} \in W_{c}, w_{n}^{2}=1, w=x_{n} w_{n} x_{n}^{-1}$, and $S_{n}=\operatorname{supp}\left(w_{n}\right)$. We will also have $S_{N} \in P$.

Let $w_{0}=w, x_{0}=1$, and let $S_{0}=\operatorname{supp}(w)$.

Assume we have constructed $\left(w_{k}, x_{k}, S_{k}\right)$ for all $k<n$. Assume further that $S_{k} \notin P$. Consider any generator $s \in S_{n-1}$ such that $s w_{n-1}<w_{n-1}$. Since $w_{n-1}^{2}=1$, we must have $w_{n-1} s<w_{n-1}$ as well. Now, if there occurs a generator $t$ in $w_{n-1}$ which does not commute with $s$, then there must be at least two occurrences of $s$ in $w_{n-1}$. Otherwise, there is exactly one occurrence of $s$ in $w_{n-1}$.

In the first case, $s w_{n-1} s$ is an involution of length $l\left(w_{n-1}\right)-2$. Let $w_{n}=$ $s w_{n-1} s$ and let $S_{n}$ be the support of $w_{n}$. Let $x_{n}=x_{n-1} s$. Note that $x_{n} w_{n} x_{n}^{-1}=$ $x_{n-1} s w_{n} s x_{n-1}^{-1}=x_{n-1} w_{n-1} x_{n-1}^{-1}=w$. Also note that $l(w)=l\left(w_{n}\right)+2 l\left(x_{n}\right)$.

In the second case, $s$ commutes with every generator appearing in $w_{n-1}$. In this case, $s$ belongs to a connected component of $S_{n-1}$ which consists of a single node. We reject this $s$ and pick another. (Recall that we are assuming that $S_{n-1} \notin P$.)

Continue in this manner until we arrive at some $S_{N} \in P$.

We then have $w_{N}=i\left(S_{N}\right)$ and $w=x_{N} w_{N} x_{N}^{-1}$ reduced, as desired.

To prove uniqueness, suppose $w=x i(S) x^{-1}$. We prove that $x=x_{N}$, and hence, $S=S_{N}$. If $x_{N}=1$, there is nothing to prove, for then $\operatorname{supp}(w) \in P$, whereas $\operatorname{supp}\left(x i(S) x^{-1}\right) \in P$ if and only if $x=1$.

Let $y_{k}=x_{k}^{-1} x$.

We claim that $l\left(y_{k}\right)=l(x)-k$ and $y_{k} i(S) y_{k}^{-1}=\left(x_{k}^{-1} x_{N}\right) w_{N}\left(x_{k}^{-1} x_{N}\right)^{-1}$.

We prove this by induction on $k$. For $k=1$, let $s=x_{1}$. Note that $l\left(s x_{N}\right)<l\left(x_{N}\right)$ and $l(s w s)=l(w)-2$. We claim that $l(s x)<l(x)$. If not, since $l(s w)<l(w)$, we know that $s$ must commute with the support of $x$ and hence, also the support of $x^{-1}$. But then, $\operatorname{sxi}(S) x^{-1} s=x \operatorname{si}(S) s x^{-1}$. We must therefore have $s \in S$. But then $s i(S) s=i(S)$ contrary to the hypothesis that $l(s w s)=l(w)-2$. We thus have $s w s=(s x) i(S)(s x)^{-1}=\left(s x_{N}\right) w_{N}\left(s x_{N}\right)^{-1}$ and the claim holds for $k=1$.

Now suppose $l\left(y_{k}\right)=l(x)-k$ for $k<n$. Note that $x_{n-1}^{-1} x_{n}$ is a simple generator; we denote it $s$. Since $l\left(s x_{n-1}^{-1} x_{N}\right)<l\left(x_{n-1}^{-1} x_{N}\right)$, we know that $s$ shortens $y_{n}$, for if not, $s y_{n} i(S) y_{n}^{-1} s$ has length no less than $y_{n} i(S) y_{n}^{-1}$, a contradiction. Thus, the claim holds for $k=n$. 
By induction, the claim holds for $k=N$. In particular, $y_{N} i(S) y_{N}^{-1}=w_{N}$. Since $y_{N} i(S) y_{N}^{-1}$ is reduced, its support consists of mutually commuting generators. We conclude that $y_{N}=1$, so that $x=x_{N}$ and $i(S)=w_{N}$ as desired.

Theorem 3.1.1 follows.

\subsection{Palindromes.}

Lemma 3.2.1. Let $s_{1}, s_{2}, s_{3}, \ldots, s_{n}$ be any sequence of generators. Let $x_{k}=$ $b_{s_{1}} b_{s_{2}} b_{s_{3}} \cdots b_{s_{k}}$ and let $y_{k}=b_{s_{k}} b_{s_{k-1}} b_{s_{k-2}} \cdots b_{s_{1}}$. Then $x_{n} y_{n}=q_{2}{ }^{m} b_{d}$ where $d^{2}=1$ and $m$ is a nonnegative integer.

Proof. We prove this by induction on $n$. When $n=1$, it follows from the relation $b_{s}^{2}=q_{2} b_{s}$.

Assume the lemma is true for all $n<N$. We prove the lemma for $n=N$. By induction, we know $x_{n-1} y_{n-1}=q_{2}{ }^{p} b_{d^{\prime}}$ where $\left(d^{\prime}\right)^{2}=1$ and $p$ is a nonnegative integer. Let $s \in \Gamma_{g}$. Define $d$ by $b_{s} b_{d^{\prime}} b_{s}=q_{2}^{r} b_{d}$. If we can show that $d^{2}=1$, then the lemma will follow.

Let $d^{\prime}=x i(S) x^{-1}$ as in theorem 3.1.1.

If $s d^{\prime}$ is not reduced, then $d=d^{\prime}$, and there is nothing to prove.

If $s d^{\prime}$ is reduced and in $W_{c}$, we have three cases: (1) either $s d^{\prime} s$ is not reduced, (2) $s d^{\prime} s$ is reduced and in $W_{c}$, or (3) $s d^{\prime} s$ is reduced but not in $W_{c}$. In case $1, s$ must commute with the support of $d^{\prime}$ so that $d=x i(S \cup\{s\}) x^{-1}=s d^{\prime}$. In case 2, $d=s d^{\prime} s$. In case $3, s$ commutes with the support of $x$, for otherwise, one of our assumptions (either $s d^{\prime} s \notin W_{c}$, or $s d^{\prime} \in W_{c}$ ) must fail. Therefore, there exists $t \in S$ such that $s i(S) s=i((S \backslash\{t\}) \cup\{s\})$ and we can apply induction to $b_{x} b_{s} b_{i(s)} b_{s} b_{x^{-1}}$.

If $s d^{\prime}$ is reduced, but not in $W_{c}$, then there exists $t \in \operatorname{supp}(x)$ which does not commute with $s$. By symmetry, $b_{s} b_{d^{\prime}} b_{s}$ can then be written as $b_{y} b_{i(S)} b_{y^{-1}}$ for some $y$ with $l(y)<N$. We can then apply induction.

We have exhausted the possibilities and the lemma follows.

\section{LEFT, RIGHT, AND TWO-SIDED CELLS}

4.1. Definition. We now imitate the definitions of left, right, and two-sided cells given by Kazhdan and Lusztig in [11]. Because of the simplicity of the structure constants for the basis $\left\{b_{w}\right\}$, the definitions of the various cells is considerably simpler than that in [11].

For any $w, w^{\prime} \in W_{c}$, we say that $w^{\prime} \leq_{L} w$ if there exists $b_{x}$ such that $b_{x} b_{w}=c b_{w^{\prime}}$ where $c \neq 0$.

For any $w, w^{\prime} \in W_{c}$, we say that $w^{\prime} \leq_{R} w$ if there exists $b_{x}$ such that $b_{w} b_{x}=c b_{w^{\prime}}$ where $c \neq 0$.

For any $w, w^{\prime} \in W_{c}$, we say that $w^{\prime} \leq_{L R} w$ if there exist $b_{x}, b_{y}$ such that $b_{x} b_{w} b_{y}=c b_{w^{\prime}}$ where $c \neq 0$.

We say $w \sim_{L} w^{\prime}$ if both $w^{\prime} \leq_{L} w$ and $w \leq_{L} w^{\prime}$. Similarly, we define $w \sim_{R} w^{\prime}$ and $w \sim_{L R} w^{\prime}$.

We shall see later (proposition 5.4.1) that in these simply laced cases, we can insist that $c=1$ in these definitions.

The equivalence classes of $\sim_{L}, \sim_{R}$, and $\sim_{L R}$ are respectively, the left, right, and two-sided cells of $W_{c}$. Define $L(w)=\left\{w^{\prime} \in W_{c} \mid w^{\prime} \sim_{L} w\right\}, R(w)=\left\{w^{\prime} \in W_{c} \mid\right.$ $\left.w^{\prime} \sim_{R} w\right\}$, and $T(w)=\left\{w^{\prime} \in W_{c} \mid w^{\prime} \sim_{L R} w\right\}$.

In general, these cells do not agree with the Kazhdan-Lusztig cells, although they do in type $A$ (see [5]). 
4.2. Multiplication in the algebra. We prove a number of facts about the algebra multiplication.

Lemma 4.2.1. Let $S \in P$. Let $s_{k}$ and $t_{k}$ be sequences of generators. Define $w$ by $b_{s_{a}} \cdots b_{s_{1}} b_{i(S)} b_{t_{1}} \cdots b_{t_{b}}=c b_{w}$. Then there exists $T \in P$ such that $T \sim S$ and some reduced expression for $w$ involves a substring of the form $i(T)$.

Proof. Let $w^{\prime}=x i(S) y$ be reduced and $s \in \Gamma_{g}$. Define $w^{\prime \prime}$ by $c b_{w^{\prime \prime}}=b_{s} b_{w^{\prime}}$.

If $s w^{\prime} \in W_{c}$ then either $w^{\prime \prime}=w^{\prime}$ or $w^{\prime \prime}=s w^{\prime}$ depending on whether $s$ shortens or lengthens $w^{\prime}$, respectively. In either case, $w^{\prime \prime}$ has a reduced expression with a substring of the form $i(S)$.

Assume $s w^{\prime} \notin W_{c}$.

If $s$ commutes with all generators in $\operatorname{supp}(x i(S))$, then $b_{s} b_{w^{\prime}}=b_{x} b_{i(S)} b_{s} b_{y}$. Note that $b_{s} b_{y}$ may be written as a product of $l(y)-1$ basis elements $b_{s_{k}}$ for $s_{k} \in \Gamma_{g}$.

If $s$ commutes with all generators in $\operatorname{supp}(x)$, but not with all the generators in $S$, then we must have $y=s y^{\prime}$ and $s$ commutes with all but one $t \in S$. Let $T=(S \cup\{s\}) \backslash\{t\}$. Observe that $T \sim S$. We compute that $b_{s} b_{w^{\prime}}=b_{x} b_{i(T)} b_{y^{\prime}}$.

If $s$ does not commute with all the generators in $\operatorname{supp}(x)$, then $b_{s} b_{x}$ can be written as a product of $l(x)-1$ basis elements $b_{s_{k}}$ for $s_{k} \in \Gamma_{g}$.

By symmetry and induction on the length of the sequence $s_{k}$, these observations imply the lemma.

We have the following corollary of lemma 4.2.1.

Corollary 4.2.2. Let $w \in W_{c}, s \in \Gamma_{g}$. Define $w^{\prime}$ by $q_{2}{ }^{m} b_{w^{\prime}}=b_{w} b_{s}$. Then $a\left(w^{\prime}\right) \geq a(w)$.

Lemma 4.2.1 can be strengthened in the following situation.

Lemma 4.2.3. Let $S \in P$. Let $s_{1}, s_{2}, s_{3}, \ldots, s_{n}$ be any sequence of generators. Define $w^{\prime}$ by $b_{i(S)} b_{s_{1}} b_{s_{2}} b_{s_{3}} \cdots b_{s_{n}}=c b_{w^{\prime}}$. Then $S \subset \mathcal{L}\left(w^{\prime}\right)$.

Proof. We proceed by induction on $n$, the case where $n=0$ being clear.

Suppose the lemma is true for all $n<N$. We prove the result holds for $n=N$.

By induction, there exists $y$ such that $b_{i(S)} b_{s_{1}} b_{s_{2}} b_{s_{3}} \cdots b_{s_{n-1}}=c^{\prime} b_{y}$ and $S \subset$ $\mathcal{L}(y)$. Define $w^{\prime}$ by $b_{w^{\prime}}=c b_{y} b_{s_{n}}$.

There are three cases to consider. Either $(1) l\left(y s_{n}\right)<l(y),(2) l\left(y s_{n}\right)>l(y)$ and $y s_{n} \in W_{c}$, or $(3) y s_{n} \notin W_{c}$.

In case $1, c=q_{2}$ and $w^{\prime}=y$, and the lemma holds.

In case $2, c=1$ and $w^{\prime}=y s_{n}$, and the lemma holds.

In case 3 , let $w_{1} s_{n} t w_{2}$ be the decomposition of $y$ as in property R5. We may further assume that $S \subset \mathcal{L}\left(w_{1} s_{n}\right)$ by commuting to the left of $s_{n} t$ all possible $s \in \operatorname{supp}\left(w_{2}\right)$. We have $b_{w_{1}} b_{s_{n}} b_{t} b_{w_{2}} b_{s_{n}}=b_{w_{1}} b_{s_{n}} b_{w_{2}}$. Although the product $w_{1} s_{n} w_{2}$ is not necessarily reduced, it represents a product of generators of length less than $N+\# S$. Thus, the lemma follows by induction.

We shall also need the following lemma related to lemma 4.2.3. In order to state and prove it, we find the following definition convenient.

Definition 4.2.4. Let $w \in W_{c}$. Let $s \in \mathcal{L}(w)$. Suppose that there exists $t \in \Gamma_{g}$ such that $s t s=t s t$ and $b_{t} b_{w}=b_{s w}$. We shall then say that $s$ can be left cancelled, or that $s$ is left cancellable, and that $s$ is left cancellable by $t$.

We make an analogous definition for $s \in \mathcal{R}(w)$. 
Lemma 4.2.5. Let $w=x i(S)$ with $a(w)=\# S$. Assume that no element of $S=$ $\mathcal{R}(w)$ is right cancellable. Let $s_{k}$ be a sequence of generators. Define $w^{\prime} \in W_{c}$ by $q_{2}{ }^{m} b_{w^{\prime}}=b_{w} b_{s_{1}} b_{s_{2}} b_{s_{3}} \cdots b_{s_{n}}$. If $a\left(w^{\prime}\right)=\# S$, then there exists $y \in W_{c}$ such that $w^{\prime}=x i(S)$ y reduced.

Proof. We prove this by induction on $n$, the case where $n=0$ being clear.

Suppose the lemma is true for $n<l$. Let $v \in W_{c}$ be defined by $q_{2}{ }^{m^{\prime}} b_{v}=$ $b_{w} b_{s_{1}} b_{s_{2}} b_{s_{3}} \cdots b_{s_{l-1}}$. If $a(v) \neq \# S$, then $a(v)>\# S$ and by corollary 4.2 .2 , this would imply that $a\left(w^{\prime}\right)>\# S$, so that there would be nothing to prove.

So assume that $a(v)=\# S$. Then by induction, there exists $y^{\prime} \in W_{c}$ such that $v=x i(S) y^{\prime}$ reduced. Define $u$ by $q_{2}{ }^{j} b_{u}=b_{v} b_{s_{l}}$. It suffices to show that $u=x i(S) y^{\prime \prime}$ reduced, for some $y^{\prime \prime} \in W_{c}$.

To see this, we consider the standard cases. If $v s_{l} \in W_{c}$, then $u=v$ or $u=v s_{l}$ depending on whether $s_{l}$ makes $v$ shorter or longer, respectively. In either case, there is nothing to show.

So assume $v s_{l} \notin W_{c}$. In this case, write $v=x_{1} s_{l} x_{2} t x_{3}$ as in property $\mathrm{R} 4^{\prime}$. Observe that $t \notin S$ since by assumption, no element of $\mathcal{R}(w)=S$ is right cancellable. On the other hand, if $t \in \operatorname{supp}(x)$ and $t \notin \operatorname{supp}\left(y^{\prime}\right)$, we must have, by property R4 $4^{\prime}$, that $s_{l}$ commutes with all generators in $S$. Consequently $a\left(w^{\prime}\right)>\# S$, and there is nothing to prove. The only remaining possibility is that $t \in \operatorname{supp}\left(y^{\prime}\right)$ so that $b_{v} b_{s_{l}}=b_{x_{1}} b_{s_{l}} b_{x_{2}} b_{x_{3}}$ and since $x_{1} s_{l} x_{2}$ can be written $x i(S) y^{\prime \prime}$ for some $y^{\prime \prime} \in W_{c}$, we can apply induction and the lemma follows.

4.3. Cancellability. This section is concerned with cancellability (definition 4.2.4).

Lemma 4.3.1. Let $w \in W_{c}$. Suppose that no element of $\mathcal{L}(w)$ or $\mathcal{R}(w)$ can be left or right cancelled, respectively. Then $w=i(S)$ for some $S \in P$.

Proof. Let $w \in W_{c}$ be as in the hypothesis of the lemma. Let $T=\{s \in \mathcal{L}(w) \mid s t=$ $t s$ for all $t \in \operatorname{supp}(w)\}$. Let $w^{\prime}=i(T) w$. If $w^{\prime}=1$ then $w=i(T)$, as desired.

So assume $w^{\prime} \neq 1$. Observe that no element of $\mathcal{L}\left(w^{\prime}\right)$ or $\mathcal{R}\left(w^{\prime}\right)$ can be cancelled. Write $w^{\prime}=i\left(\mathcal{L}\left(w^{\prime}\right)\right) x i\left(\mathcal{R}\left(w^{\prime}\right)\right)$ reduced. Let $y=i\left(\mathcal{L}\left(w^{\prime}\right)\right) x i\left(\mathcal{R}\left(w^{\prime}\right)\right) x^{-1}$. We claim that $l\left(y^{n}\right)=n l(y)$ and that $y^{n} \in W_{c}$.

To see this, consider the sequence of generators obtained by repeating the sequence which begins with the generators in $\mathcal{L}\left(w^{\prime}\right)$, followed by a reduced expression for $x$, followed by the generators in $\mathcal{R}\left(w^{\prime}\right)$, followed by a reduced expression for $x^{-1}$. By property R3, if between any two consecutive occurrences of a generator $s \in \Gamma_{g}$ in this sequence, there occurs at least two generators which do not commute with $s$, our claim would follow.

The only non-trivial case to consider is where one $s$ appears in a reduced expression for $x$ and the other $s$ appears in a reduced expression for $x^{-1}$. However, since no element of $\mathcal{L}\left(w^{\prime}\right)$ or $\mathcal{R}\left(w^{\prime}\right)$ can be cancelled, we conclude that there must be at least two occurrences of generators between the two occurrences of $s$, which do not commute with $s$. (There cannot be 0 such generators because $w^{\prime}=i\left(\mathcal{L}\left(w^{\prime}\right)\right) x i\left(\mathcal{R}\left(w^{\prime}\right)\right)$ is reduced.)

This contradicts finiteness of $W_{c}$ (see, for instance, [3, prop. 2], or lemma 8.1.4).

Lemma 4.3.2. Let $w=i(S) x \in W_{c}$. Assume that $a(w)=\# S$ and that no element of $\mathcal{L}(w)$ can be left cancelled. Write $w=i\left(T_{1}\right) \cdots i\left(T_{m}\right)$ reduced, where $T_{k}=$ $\mathcal{L}\left(i\left(T_{k-1}\right) \cdots i\left(T_{1}\right) w\right) \in P$ and $T_{m} \neq \emptyset$. Let $\gamma_{k}, 1 \leq k<m$, denote the subgraph 
of $\Gamma$ which contains the nodes in $T_{k}$ and $T_{k+1}$ along with all edges whose endpoints are in $T_{k} \cup T_{k+1}$. Then no connected component of $\gamma_{k}$ has more nodes in $T_{k+1}$ than in $T_{k}$.

Proof. We shall prove this by induction on the length of $x$.

First, note that $\mathcal{L}(w)=S=T_{1}$. Suppose $s \in \mathcal{L}(w)$ commutes with $\operatorname{supp}(w)$. Then $a(s w)=a(w)-1$ and $s w$ satisfies the hypothesis of the lemma. By induction, we have the result for $s w$, and one can see this implies the result for $w$. So we assume that no $s \in \mathcal{L}(w)$ commutes with $\operatorname{supp}(w)$.

Consider the element $w^{\prime}=i\left(T_{1}\right) \cdots i\left(T_{m-1}\right)$. Then $w^{\prime}$ satisfies the conditions of the lemma, and we conclude that $\gamma_{k}$ contains no connected component with more nodes in $T_{k+1}$ than in $T_{k}$ for $k<m-1$.

Thus, if there is a connected component $C$ which contradicts the conclusion of the lemma, then it must occur in $\gamma_{m-1}$.

Let $s \in \mathcal{R}(w)$. Define $T_{k}^{\prime}$ and $\gamma_{k}^{\prime}$ for $w s$ in the same way $T_{k}$ and $\gamma_{k}$ are defined for $w$. Note that $w s$ satisfies the hypothesis of the lemma and has shorter length than $w$. By induction, the lemma holds for $w s$.

If $s$ does not correspond to a node in $C$, then $C$ still exists as a connected component of $\gamma_{m-1}^{\prime}$ for $w s$, contradicting the inductive hypothesis.

Thus, we can assume that $C$ involves all the nodes of $T_{m}$ and $T_{m}=\mathcal{R}(w)$. But this implies that $C=\gamma_{m-1}$.

If $C$ forms a subgraph of $\Gamma$ of type $A$, then the only way $C$ can have more nodes in $T_{m}$ is if every node of $T_{m-1}$ is connected to two nodes of $T_{m}$. But this implies that no element of $\mathcal{R}(w)$ can be right cancelled. This would contradict lemma 4.3.1.

So we can assume that $C$ forms a subgraph not of type $A$. In type $D$ or $E$, there exists a node of $\Gamma$ whose removal results in a graph of type $A$. Call this node 0 . Thus, $C$ involves the node 0 .

Let $C^{\prime}$ be the subgraph of $C$ obtained by removing the 0 node.

If $0 \in T_{m-1}$, then $C^{\prime}$ has at least 2 more nodes in $T_{m}$ than in $T_{m-1}$. However, since $C^{\prime}$ is a graph of type $A$, this is impossible.

Thus $0 \in T_{m}$. Since $C$ is not of type $A$, we must have the branch node in $T_{m-1}$ and we must have all nodes connected to the branch node (including 0 ) in $T_{m}$. Let $w^{\prime}=w 0$. Then $w^{\prime}$ satisfies the conditions of the lemma and if one defines $T_{k}^{\prime}$ for $w^{\prime}$ as $T_{k}$ is defined for $w$, we see that $T_{k}^{\prime}=T_{k}$ for $k<m$ and $T_{m}=T_{m}^{\prime} \cup\{0\}$. By induction, we conclude that if $C$ has more nodes in $T_{m}$ than in $T_{m-1}$, then $C^{\prime}$ has the same number of nodes in $T_{m}$ and $T_{m-1}$. By lemma 4.3.1, there must be a node $t \in T_{m-1}$ which is connected to a unique $s \in T_{m}$. (Recall that all nodes in $\Gamma$ have degree 1 or 2 , with the exception of the branch node, which we know belongs to $T_{m-1}$.) Now $s \neq 0$ since the branch generator is connected to three generators in $T_{m}$. Removal of $s$ from $C$ results in two subgraphs: an isolated point consisting of $t$ and a graph $C^{\prime \prime}$ consisting of the nodes $C \backslash\{s, t\}$. Since $s \in T_{m}$ and $t \in T_{m-1}$, $C^{\prime \prime}$ has more nodes in $T_{m}$ than in $T_{m-1}$ as well. However, the induction hypothesis applied to $w s$ rules this possibility out.

The lemma follows.

We remark that the hypothesis that no element of $\mathcal{L}(w)$ can be left cancelled is necessary. For instance, in $E_{5}=D_{5}$, if we label the the generators so that 1,2 , 3 , and 4 naturally correspond to a subgraph of type $A_{4}$, with 3 being the branch node and 0 the remaining generator, then $w=14302$ provides a counterexample. 
We also wish to point out that the theorem is not true in type $\hat{D}_{4}$, for if we label the generators so that 3 is the branch node and $A, B, C$, and $D$ are the other nodes, then $w=A B 3 C D$ satisfies the hypothesis of the lemma, but not the conclusion.

Lemma 4.3.3. In the same setup as lemma 4.3.2, there must exist some element of $T_{m}$ which is right cancellable by an element of $T_{m-1}$.

Proof. We know that no connected component $C$ of $\gamma_{m-1}$ has more nodes in $T_{m}$ than in $T_{m-1}$.

If no element of $C \cap T_{m}$ were right cancellable, then every element of $C \cap T_{m-1}$ must be connected to at least two nodes of $C \cap T_{m}$. This means there are at least $2 \# C \cap T_{m-1}$ edges in $C$. Since there is only one branch node, this implies the existence of at least $\left(2 \# C \cap T_{m-1}-1\right) / 2=\# C \cap T_{m-1}-1 / 2$ nodes in $C \cap T_{m}$. Consequently, the number of nodes in $C$ must be $2 \# C \cap T_{m-1}$. If each node in $C$ has degree 2 , then $C$ is a cycle, in other words, a graph of type affine $A$. Otherwise, the branch node must occur in $C \cap T_{m}$ and it must be connected to all its three neighbors in $\Gamma$. However, at least one of these neighbors commutes with every generator except the branch generator, and this implies that the branch generator is cancellable. In either case, we have found a node in $C \cap T_{m}$ which is right cancellable by an element of $C \cap T_{m-1}$. The lemma follows.

Proposition 4.3.4. In the same setup as lemma 4.3.2, we have $a\left(i\left(T_{1}\right) w\right)=\# T_{2}$.

Proof. Repeated application of lemma 4.3.3 implies that there exists $w^{\prime}$ such that $b_{i\left(T_{2}\right)}=b_{i\left(T_{1}\right) w} b_{w^{\prime}}$. The proposition follows.

4.4. Right cells. We are now ready to begin classifying right cells.

Lemma 4.4.1. Let $w \in W_{c}$. Write $w=i(S) x$ reduced, where $S \in P$. Assume that $a(w)=\# S$ and $l(x) \geq 1$. Then there exists $s \in \mathcal{R}(w)$ which can be right cancelled.

Proof. We shall prove this by induction on the length of $x$.

When $x \in \Gamma_{g}$, we see that $x$ cannot commute with all of the generators in $S$ since $a(w)=\# S$. Let $t$ be a generator in $S$ which does not commute with $x$. Then $x$ is right cancellable by $t$.

Now assume the lemma is true for all $x$ with $l(x)<l$. We prove the lemma for $x$ of length $l$.

If no member of $\mathcal{R}(w)$ is right cancellable, then by lemma 4.3.1, there must be an element $s^{\prime} \in \mathcal{L}(w)$ such that $s^{\prime}$ is left cancellable, say by $t^{\prime}$. Thus, $b_{t^{\prime}} b_{w}=b_{s^{\prime} w}$. Note that $t^{\prime}$ commutes with every generator in $S$ except for $s^{\prime}$. Let $S^{\prime}=\left(S \backslash\left\{s^{\prime}\right\}\right) \cup\left\{t^{\prime}\right\}$. Then $s^{\prime} w=i\left(S^{\prime}\right) x^{\prime}$ reduced where $x^{\prime}=t^{\prime} x$. Since $\# S=a(w) \geq a\left(s^{\prime} w\right) \geq \# S^{\prime}=$ $\# S$, we have $a\left(s^{\prime} w\right)=\# S$. Therefore, by induction, there exists $s \in \mathcal{R}\left(s^{\prime} w\right)$ which can be right cancelled, unless $x^{\prime}=1$. If $x^{\prime} \neq 1$, write $s^{\prime} w=x_{1} t s x_{2}$ as in property $\mathrm{R}^{\prime}$. Then $w=s^{\prime} x_{1} t s x_{2}$ and this shows that $s$ is right cancellable in $w$ as well.

If $x^{\prime}=1$, then $x \in \Gamma_{g}$, and we have seen that this means $x$ itself is right cancellable.

Either way, the lemma follows.

Proposition 4.4.2. Let $w \in W_{c}$. Suppose there exists $S \in P$ and $x \in W_{c}$ such that $w=i(S) x$ is reduced. Assume that $a(w)=\# S$. Then $w \sim_{R} i(S)$. Furthermore, there exists $w^{\prime} \in W_{c}$ such that $b_{i(S)}=b_{w} b_{w^{\prime}}$. 
Proof. We have $b_{w}=b_{i(S)} b_{x}$ so that $w \leq_{R} i(S)$. So we show that $i(S) \leq_{R} w$.

Let $s$ and $t$ be as in the proof of lemma 4.4.1. Write $w=w_{1} t s w_{2}$ as in property R5. By uniqueness of $s$ in this expression, we see that $s$ commutes with every generator in the support of $w_{2}$. We compute that $b_{w} b_{t}=b_{w_{1}} b_{t} b_{s} b_{w_{2}} b_{t}=$ $b_{w_{1}} b_{t} b_{s} b_{t} b_{w_{2}}=b_{w_{1}} b_{t} b_{w_{2}}$. Since $s \in \mathcal{R}(w)$, we have $w_{1} t w_{2}$ is reduced. By lemma 4.2.3, $w_{1} t w_{2}=i(S) x^{\prime}$ where $l\left(x^{\prime}\right)=l(x)-1$. Thus, $w_{1} t w_{2} \leq_{R} w$. Therefore, by induction on $l(x)$, we have $i(S) \leq_{R} w$.

Indeed, it follows that we can construct a sequence $t_{1}, t_{2}, \ldots, t_{n}$ where $n=l(x)$ and $b_{i(S)}=b_{w} b_{t_{1}} \cdots b_{t_{n}}$. By positivity, it follows that $b_{t_{1}} \cdots b_{t_{n}}=b_{w^{\prime}}$ for some $w^{\prime} \in W_{c}$.

Proposition 4.4.3. Let $S \in P$. Let $R=\left\{w \in W_{c} \mid w=i(S) x, a(w)=\# S\right\}$. Then $R$ is a right cell.

Proof. This follows from lemma 4.2.3 and proposition 4.4.2.

The proof of the following theorem requires use of proposition 5.3.1 and lemma 5.3.2. We have been careful to check that we are not creating a circular argument.

Theorem 4.4.4. The set of right cells are in bijection with the set of involutions in $W_{c}$. The bijection is given by $d \mapsto R(d)$, where $d^{2}=1$.

Proof. First we show that for every $w \in W_{c}$, there exists an involution $d \in W_{c}$ such that $w \sim_{R} d$.

Write $w=x i(S) y$ so that $a(w)=\# S$. Assume further that $x i(S)$ cannot be written $x_{1} i(T) x_{2}$ reduced, where $x_{2} \neq 1$ and $T \sim S$. By proposition 4.4.2 and its dual, there exist $x^{\prime}, y^{\prime} \in W_{c}$ such that $b_{x^{\prime}} b_{x i(S)}=b_{i(S)}$ and $b_{i(S) y} b_{y^{\prime}}=b_{i(S)}$. In particular, $b_{w} b_{y^{\prime}}=b_{x i(S)}$, so that $x i(S) \leq_{R} w$. On the other hand $b_{x i(S)} b_{y}=b_{w}$ so that $w \leq_{R} x i(S)$. Thus, $x i(S) \sim_{R} w$.

By lemma 3.2.1, $b_{x i(S)} b_{x^{-1}}=q_{2}{ }^{-\# S} b_{x i(S)} b_{i(S)} b_{x^{-1}}=q_{2}{ }^{m} b_{d}$ for some involution $d$. On the other hand, $q_{2}{ }^{m} b_{d} b_{x^{\prime-1}}=b_{x} b_{i(S)} b_{x^{-1}} b_{x^{\prime-1}}=b_{x} b_{i(S)}$. We conclude that $d \sim_{R} x i(S)$, whence $d \sim_{R} w$.

By lemma 5.3.2, if $d$ and $d^{\prime}$ are involutions such that $d \sim_{R} d^{\prime}$, then $d=d^{\prime}$. Theorem 4.4.4 follows.

Because of the symmetry of the defining relations, each statement we have made about right cancellability or right cells has a dual counterpart for left cancellability and left cells.

\subsection{Two-sided cells.}

Theorem 4.5.1. The two-sided cells are in bijective correspondence with the set $\bar{P}$. The bijection is given by $\bar{S} \mapsto T(i(S))$.

Proof. We begin with the following lemma.

Lemma 4.5.2. We have $S \sim T$ if and only if $i(S) \sim_{L R} i(T)$.

Proof. First we show that $S \sim T$ implies $i(S) \sim_{L R} i(T)$. It suffices to check this in the case where $S$ and $T$ are neighbors.

Define $s$ and $t$ by $S=(S \cap T) \cup\{s\}$ and $T=(S \cap T) \cup\{t\}$. Observe that $b_{i(S)}=b_{s} b_{i(T)} b_{s}$ and $b_{i(T)}=b_{t} b_{i(S)} b_{t}$.

For the other implication, suppose $S \nsim T$. By lemma 4.2.1, every $w$ such that there exists a constant $c$ for which $c b_{w}=b_{x} b_{i(S)} b_{y}$ must have a reduced expression 
which involves a substring of the form $i\left(S^{\prime}\right)$ for some $S^{\prime} \sim S$. Consequently, we cannot have $i(S) \sim_{L R} i(T)$.

We now return to the proof of theorem 4.5.1.

In view of lemma 4.5.2, it suffices to show that every $w \in W_{c}$ satisfies $w \sim_{L R} i(S)$ for some $S \in P$. Let $S$ be of cardinality $a(w)$ such that $w=x i(S) y$ reduced. By definition, $w \leq_{L R} i(S)$.

Note that $a(x i(S))=a(i(S) y)=a(w)$. By proposition 4.4.2 and its dual, there exist $x^{\prime}, y^{\prime} \in W_{c}$ such that $b_{x^{\prime}} b_{x} b_{i(S)}=b_{i(S)}$ and $b_{i(S)} b_{y} b_{y^{\prime}}=b_{i(S)}$. Thus, $b_{x^{\prime}} b_{w} b_{y^{\prime}}=b_{i(S)}$ and $i(S) \leq_{L R} w$. The theorem follows.

\section{Semi-Simplicity}

5.1. Linear functions. Let $f: \overline{\mathcal{H}} \rightarrow \mathbb{Q}\left(q^{1 / 2}\right)$ be the $\mathbb{Q}\left(q^{1 / 2}\right)$-linear map defined by $f\left(b_{w}\right)=1$ if $w^{2}=1, f\left(b_{w}\right)=0$ if $w^{2} \neq 1$, and then extending linearly. By the symmetry of the defining relations, we have $f\left(b_{w}\right)=f\left(b_{w^{-1}}\right)$.

Define a bilinear form $():, \overline{\mathcal{H}} \times \overline{\mathcal{H}} \rightarrow \mathbb{Q}\left(q^{1 / 2}\right)$ by defining $\left(b_{w}, b_{w^{\prime}}\right)=f\left(b_{w^{-1}} b_{w^{\prime}}\right)$ and then extending linearly.

Observe that $($,$) satisfies (x, y)=(y, x)$ and $\left(b_{s} x, y\right)=\left(x, b_{s} y\right)$, where $s \in \Gamma_{g}$, $x, y \in \overline{\mathcal{H}}$.

\subsection{On the diagonal.}

Proposition 5.2.1. We have $\left(b_{w}, b_{w}\right)=q_{2}{ }^{a(w)}$.

The proof consists of a series of lemmas.

Lemma 5.2.2. Let $S \in P$. We have $b_{i(S)} b_{i(S)}=q_{2}{ }^{\# S} b_{i(S)}$.

Proof. This follows from direct multiplication.

Lemma 5.2.3. Let $w=i(S) x$ be reduced and assume $a(w)=\# S$.

We have $b_{x^{-1}} b_{i(S)} b_{x}=b_{d}$ for some involution $d$.

Proof. We proceed by induction on $l(x)$, the case where $x=1$ being clear.

By proposition 4.4.2, there exists $w^{\prime} \in W_{c}$ such that $b_{w} b_{w^{\prime}}=b_{i(S)}$. We compute that $b_{x^{-1}} b_{i(S)} b_{x} b_{w^{\prime}}=b_{x^{-1}} b_{i(S)}$. Therefore, by positivity, $b_{x^{-1}} b_{i(S)} b_{x}=b_{d}$ for some $d$. On the other hand, $b_{x^{-1}} b_{i(S)} b_{x}=q_{2}{ }^{-\# S} b_{x^{-1}} b_{i(S)} b_{i(S)} b_{x}=c b_{d^{\prime}}$ for some involution $d^{\prime}$ by lemma 3.2.1. We conclude that $d=d^{\prime}$ is an involution and the lemma follows.

As an immediate corollary, we have the following.

Corollary 5.2.4. Let $w=i(S) x$ be reduced and assume $a(w)=\# S$.

We have $b_{x^{-1}} b_{i(S)} b_{i(S)} b_{x}=q_{2}{ }^{a(w)} b_{d}$ for some involution $d$.

Lemma 5.2.5. Let $w=i(S) x$ be reduced and assume $a(w)=\# S$.

We have $b_{i(S)} b_{x} b_{x^{-1}} b_{i(S)}=q_{2}{ }^{a(w)} b_{i(S)}$.

Proof. We prove this by induction on $l(x)$.

By lemma 4.4.1, there exist $s \in \mathcal{R}(x)$ and $t \in \Gamma_{g}$ such that $b_{x} b_{t}=b_{y}$ where $y=x s$. Note that $t \in \mathcal{R}(y)$. We have

$$
\begin{aligned}
& b_{i(S)} b_{x} b_{x^{-1}} b_{i(S)}=b_{i(S)} b_{y} b_{s} b_{s} b_{y^{-1}} b_{i(S)}=q_{2} b_{i(S)} b_{y} b_{s} b_{t} b_{(y t)^{-1}} b_{i(S)} \\
& \quad=b_{i(S)} b_{y} b_{s} b_{t} b_{t} b_{(y t)^{-1}} b_{i(S)}=b_{i(S)} b_{y} b_{t} b_{(y t)^{-1}} b_{i(S)}=b_{i(S)} b_{y} b_{y^{-1}} b_{i(S)} .
\end{aligned}
$$

The lemma follows by induction and lemma 5.2.2. 
Lemma 5.2.6. We have $b_{w^{-1}} b_{w}=q_{2}{ }^{a(w)} b_{d}$ where $d^{2}=1$.

Proof. We can write $w=x i(S) y$ reduced and $a(w)=\# S$.

Write $b_{w^{-1}} b_{w}=c b_{d}$. We know from lemma 3.2.1 that $d^{2}=1$.

By proposition 4.4.2, there exists $w^{\prime}$ such that $b_{i(S) y} b_{w^{\prime}}=b_{i(S)}$. Then $b_{w} b_{w^{\prime}}=$ $b_{x i(S)}$. By lemma 5.2.5, we see that $b_{\left(w^{\prime}\right)^{-1}} b_{w^{-1}} b_{w} b_{w^{\prime}}=b_{i(S) x^{-1}} b_{x i(S)}=q_{2}{ }^{a(w)} b_{i(S)}$.

By positivity, we conclude that $c=q_{2}{ }^{a(w)}$. The lemma follows.

The proof of proposition 5.2.1 follows from lemma 5.2.6 and the definitions.

\subsection{More on involutions.}

Proposition 5.3.1. Let $d \in W_{c}$ satisfy $d^{2}=1$. Write $d=x i(S) x^{-1}$ as in theorem 3.1.1. We have $a(d)=\# S$.

Proof. Let $y=i(S) x^{-1}$. We can always write $y=u i(T) v$ reduced, where $a(y)=$ $\# T$.

We compute that $q_{2}{ }^{\# S} b_{d}=b_{y^{-1}} b_{y}=b_{v^{-1}} b_{i(T)} b_{u^{-1}} b_{u} b_{i(T)} b_{v}$. By lemma 5.2.5, we know $b_{i(T)} b_{u^{-1}} b_{u} b_{i(T)}=q_{2}^{\# T} b_{i(T)}$. Therefore, $q_{2}^{\# S} b_{d}=q_{2}^{\# T} b_{v^{-1}} b_{i(T)} b_{v}$. By lemma 5.2.3, we have $b_{v^{-1}} b_{i(T)} b_{v}=b_{d^{\prime}}$ for some involution $d^{\prime}$. This implies that $d=d^{\prime}$ and $\# S=\# T$, as desired.

We now complete the proof of theorem 4.4.4, which follows from the following lemma.

Lemma 5.3.2. Let $d$ and $d^{\prime}$ be involutions such that $d \sim_{R} d^{\prime}$. Then $d=d^{\prime}$.

Proof. Assume $d \neq d^{\prime}$.

Write $d=x i(S) x^{-1}$ and $d^{\prime}=y i(T) y^{-1}$ as in theorem 3.1.1. By proposition 5.3.1, we know that $a(d)=\# S$ and $a\left(d^{\prime}\right)=\# T$. By the dual to proposition 4.4.2, there exist $x^{\prime}$ and $y^{\prime}$ such that $b_{i(S) x^{-1}} b_{x^{\prime}}=b_{i(S)}$ and $b_{i(T) y^{-1}} b_{y^{\prime}}=b_{i(T)}$. Thus, $x i(S) \sim_{R} d$ and $y i(T) \sim_{R} d^{\prime}$. It suffices to show that $x i(S) \chi_{R} y i(T)$.

Suppose to the contrary that $x i(S) \sim_{R} y i(T)$. Then there exists $v$ such that $b_{x i(S)} b_{v}=q_{2}^{m} b_{y i(T)}$.

Note that no element of $\mathcal{R}(x i(S))=S$ can be right cancelled since $x i(S) x^{-1} \in$ $W_{c}$. Applying lemma 4.2.5, it follows that $y i(T)=x i(S) x_{1}$ for some $x_{1} \in W_{c}$ such that $x i(S) x_{1}$ is reduced. By symmetry, we also have $y_{1} \in W_{c}$ such that $y i(T) y_{1}$ is reduced and equal to $x i(S)$. But then $y i(T) y_{1} x_{1}$ is reduced and equal to $y i(T)$. Thus, $y_{1}=x_{1}=1$ and $y i(T)=x i(S)$. Since $\mathcal{R}(y i(T))=T$ and $\mathcal{R}(x i(S))=S$, we conclude that $y=x$. But then $d=d^{\prime}$ contrary to hypothesis. The lemma follows.

\subsection{More on structure constants.}

Proposition 5.4.1. Let $w \in W_{c}$ and $s \in \Gamma_{g}$. Define $w^{\prime}$ by $b_{s} b_{w}=q_{2}{ }^{m} b_{w^{\prime}}$ for some constant $m$. If $l(s w)<l(w)$, then $m=1, w^{\prime}=s w$. If $l(s w)>l(w)$ and $s w \in W_{c}$, then $m=0, w^{\prime}=s w$. If $s w \notin W_{c}$, then $m=0$.

Proof. We shall prove this by induction on $l(w)$, the case where $w=1$ being clear.

Suppose the proposition is true for $l(w)<n$. Let $w \in W_{c}$ be of length $n$.

If $s w \in W_{c}$ then the result of multiplying $b_{s}$ by $b_{w}$ follows from the definition of $b_{w}$ or the relation $b_{s}^{2}=q_{2} b_{s}$ depending on whether $s$ makes $w$ longer or shorter.

Now assume $s w \notin W_{c}$. Let $t$ be as in property $\mathrm{R} 5{ }^{\prime}$. 
Suppose $w=x i(S) y$ where $a(w)=\# S$. By proposition 4.4.2, there exists $y^{\prime}$ such that $b_{i(S) y} b_{y^{\prime}}=b_{i(S)}$. Since $b_{s} b_{w} b_{y^{\prime}}=b_{s} b_{x i(S)}$, we can assume that $y=1$, for otherwise our induction hypothesis implies the proposition.

Using the dual to lemma 4.4.1, there exists $u \in \Gamma_{g}$ such that $b_{u} b_{w}=b_{v}$ where $w=s^{\prime} v, s^{\prime}$ and $u$ do not commute, and $s^{\prime} \in \mathcal{L}(w)$. Let $U$ denote the set of such $u \in \Gamma_{g}$.

If $t \in \mathcal{L}(w)$, then we can write $w=t s x_{2}$. In this case $b_{s} b_{w}=b_{s x_{2}}$ and the proposition follows.

Assume $t \notin \mathcal{L}(w)$.

Suppose that $s$ commutes with some element $u \in U$. In this case, $b_{u} b_{s} b_{w}=$ $b_{s} b_{u} b_{w}=b_{s} b_{v}$ where $w=s^{\prime} v$ and $s^{\prime} \in \mathcal{L}(w)$. Since $t \notin \mathcal{L}(w)$, we know $t \neq s^{\prime}$. Hence, the induction hypothesis implies $b_{s} b_{v}=b_{w^{\prime \prime}}$ for some $w^{\prime \prime} \in W_{c}$. This implies that $b_{s} b_{w}=b_{w^{\prime}}$ for some $w^{\prime} \in W_{c}$ by positivity of structure constants (recall that $\left.b_{u} b_{s} b_{w}=b_{w^{\prime \prime}}\right)$.

So assume now that $s$ does not commute with any $u \in U$. We claim that we must then have $U=\{t\}$. To see this, let $u \in U$. Note that $u \in \operatorname{supp}(w)$. Let $s^{\prime} \in \mathcal{L}(w)$ be such that $b_{u} b_{w}=b_{s^{\prime} w}$. Write $w=x_{1} s^{\prime} u x_{2}$ as in property R $5^{\prime}$. Since $u$ commutes with $\operatorname{supp}\left(x_{1}\right)$ and since $s^{\prime} \in \mathcal{L}(w)$, we can in fact assume $x_{1}=1$. Since $t \notin \mathcal{L}(w)$, we know $t \neq s^{\prime}$. If $t \neq u$, then $t \in \operatorname{supp}\left(x_{2}\right)$. But then before the first occurrence of $s$ in $w$ there occurs a $t$ and a $u$, contradicting the assumption that $s w \notin W_{c}$. Therefore, we must have $u=t$.

We conclude that $w=s^{\prime} t s x^{\prime}$ reduced. Thus $b_{s} b_{w}=b_{s^{\prime}} b_{s x^{\prime}}$. To complete the argument it suffices, by induction, to show that $s^{\prime} \notin \mathcal{L}\left(x^{\prime}\right)$. This follows since we know $s s^{\prime}=s^{\prime} s$ and if $s^{\prime} \in \mathcal{L}\left(x^{\prime}\right)$ then $w=s^{\prime} t s s^{\prime} y^{\prime}$ reduced, which contradicts the assumption that $w \in W_{c}$.

This completes the proof of proposition 5.4.1.

We remark that proposition 5.4.1 implies (only in the simply laced case) that we can redefine $\leq_{L}$ as follows: For any $w, w^{\prime} \in W_{c}$, we have $w^{\prime} \leq_{L} w$ if and only if there exists $b_{x}$ such that $b_{x} b_{w}=b_{w^{\prime}}$. Similarly, we can redefine $\leq_{R}$ and $\leq_{L R}$. This means that the cell structure is independent of the parameter $q$.

\subsection{Off the diagonal.}

Theorem 5.5.1. Let $x, y \in W_{c}$. We claim that either $\left(b_{x}, b_{y}\right)=0$, or $\left(b_{x}, b_{y}\right)=$ $q_{2}{ }^{m}$ where $m \leq(a(x)+a(y)) / 2$, with equality if and only if $x=y$.

In fact, we shall also prove that $m \leq \min (a(x), a(y))$.

Lemma 5.5.2. Let $S, T \in P$.

We have $b_{i(S)} b_{i(T)}=q_{2}{ }^{\# S \cap T} b_{i(S)} b_{i(T \backslash S)}$.

Proof. The proof follows by direct computation.

Lemma 5.5.3. Let $S, T \in P$. Suppose $x^{\prime}=i(S) x$ and $y^{\prime}=y i(T)$ so that $a\left(x^{\prime}\right)=\# S$ and $a\left(y^{\prime}\right)=\# T$. We have $b_{x^{\prime}} b_{y^{\prime}}=q_{2}{ }^{m} b_{w}$ for some $w \in W_{c}$ and $m \leq \min (\# S, \# T)$.

Proof. First suppose that there exists $s \in \mathcal{L}\left(x^{\prime}\right)$ which can be left cancelled by $t$. We have seen that $a\left(x^{\prime}\right)=a\left(s x^{\prime}\right)$. Since $b_{x^{\prime}} b_{y^{\prime}}=b_{s} b_{t} b_{x^{\prime}} b_{y^{\prime}}=b_{s} b_{s x^{\prime}} b_{y^{\prime}}$, it suffices to prove the lemma for $s x^{\prime}$ in place of $x^{\prime}$. An analogous argument for right cancellation of elements in $\mathcal{R}\left(y^{\prime}\right)$ enables us to assume that no element of $\mathcal{L}\left(x^{\prime}\right)$ is left cancellable and no element of $\mathcal{R}\left(y^{\prime}\right)$ is right cancellable. 
Write $x^{\prime}=i\left(T_{1}\right) \cdots i\left(T_{m}\right)$ and define $\gamma_{k}$ as in the statement of lemma 4.3.2. By lemma 4.3.2, each connected component of $\gamma_{k}$ has at least as many nodes in $T_{k}$ as in $T_{k+1}$.

Define, for $1 \leq k \leq m$, the sequence of integers $m_{k}$ and the elements $w_{k} \in W_{c}$ by $q_{2}{ }^{m_{k}} b_{w_{k}}=b_{i\left(T_{k}\right) \cdots i\left(T_{m}\right)} b_{y^{\prime}}$. We set $m_{m+1}=0$.

We claim that $m_{k}-m_{k+1} \leq \# T_{k}-\# T_{k+1}$.

By lemma 4.2.3, $T_{k+1} \subset \mathcal{L}\left(w_{k+1}\right)$. Because each connected component $C$ in $\gamma_{k}$ has at least as many nodes in $T_{k}$ as in $T_{k+1}$, we can map each node $s \in T_{k+1}$ to a distinct node of $T_{k}$ which is connected to $s$. To see this, note that this is clear if $C$ is a graph of type $A$. Otherwise, $C$ contains a node, $s$, removal of which yields a subgraph $C^{\prime}$ of type $A$. If $C^{\prime}$ has more nodes in $T_{k+1}$ than in $T_{k}$, then we can obtain such a map by sending the branch node to $s$. If $C^{\prime}$ has the same number of nodes in $T_{k}$ and $T_{k+1}$, then there is one way to create the mapping for nodes in $C^{\prime}$ and since $s \in T_{k}$, this mapping works for $C$. Otherwise, $C^{\prime}$ has fewer nodes in $T_{k+1}$ than in $T_{k}$. Here, we only have to be concerned with the case $s \in T_{k+1}$. But then we see that there is a map which sends $s$ to the branch node. Let $T_{k}^{\prime} \subset T_{k}$ be the set of nodes which are mapped to in this manner. By proposition 5.4.1, there exists $w^{\prime}$ such that $b_{w^{\prime}}=b_{i\left(T_{k}^{\prime}\right)} b_{w_{k+1}}$. The claim follows.

In particular, we find that $m=m_{1} \leq \# T_{1} \leq a(\# S)$. By a dual argument, we also have $m \leq a(\# T)$. The lemma follows.

Lemma 5.5.4. Let $S, T \in P$. Suppose $x^{\prime}=i(S) x$ and $y^{\prime}=y i(T)$ so that $a\left(x^{\prime}\right)=$ $a\left(y^{\prime}\right)=\# S=\# T$. Furthermore, assume that no element of $\mathcal{L}\left(x^{\prime}\right)$ can be left cancelled and no element of $\mathcal{R}\left(y^{\prime}\right)$ can be right cancelled.

We have $b_{x^{\prime}} b_{y^{\prime}}=q_{2}{ }^{m} b_{w}$ for some $w \in W_{c}$. If $m=a\left(x^{\prime}\right)$, then $x^{\prime}=y^{\prime-1}$.

Proof. Write $x^{\prime}=i\left(T_{1}\right) \cdots i\left(T_{m}\right)$ as in the proof of lemma 5.5.3, and define $m_{k}$ and $w_{k}$ as in that proof as well.

Examining the proof of lemma 5.5.3, we see that if $m=a\left(x^{\prime}\right)$, then we must have equalities occurring wherever we wrote an inequality in that proof.

In other words, we must have $T_{m} \subset \mathcal{L}\left(y^{\prime}\right)$. Thus, $y^{\prime}=i\left(T_{m}\right) y_{m}$ reduced.

Thus $b_{x^{\prime}} b_{y^{\prime}}=q_{2} \# T_{m} b_{i\left(T_{1}\right) \cdots i\left(T_{m}\right)} b_{y_{m}}$.

Now each member $s \in T_{m}$ can be paired with a distinct member of $T_{m-1}$ which does not commute with $s$, just as in the proof of lemma 5.5.3. Again, denote by $T_{m-1}^{\prime}$ the members of $T_{m-1}$ so mapped to. In order for $m_{m-1}-m_{m}=\# T_{m-1}-$ $\# T_{m}$, we must be able to select $T_{m-1}^{\prime}$ in such a way that that $b_{i\left(T_{m-1}^{\prime}\right)} b_{i\left(T_{m}\right)} b_{y_{m}}=$ $b_{y_{m}}$. For this to be possible, we must have $T_{m-1}^{\prime} \subset \mathcal{L}\left(y_{m}\right)$. In addition, for equality to occur, we must have $T_{m-1} \backslash T_{m-1}^{\prime} \subset \mathcal{L}\left(y_{m}\right)$. Thus, we can write $y_{m}=$ $i\left(T_{m-1}\right) y_{m-1}$ reduced.

We find that $b_{x^{\prime}} b_{y^{\prime}}=q_{2}^{\# T_{m-1}} b_{i\left(T_{1}\right) \cdots i\left(T_{m-1}\right)} b_{y_{m-1}}$. We argue in a similar fashion until we find $y=i\left(T_{m}\right) i\left(T_{m-1}\right) i\left(T_{m-2}\right) \cdots i\left(T_{1}\right) y_{1}$ reduced.

Since $T_{1}=S$, we have $a\left(i\left(T_{1}\right) y_{1}\right)=\# S=\# T$. By lemma 4.4.1, this implies that some element of $\mathcal{R}\left(y_{1}\right)$ is right cancellable, unless $y_{1}=1$. By hypothesis, no element of $\mathcal{R}\left(y_{1}\right)$ is right cancellable, so we conclude that $y_{1}=1$ and $y^{\prime}=x^{\prime-1}$, as desired. The lemma follows.

We now prove theorem 5.5.1.

Let $x, y \in W_{c}$. We can always write $x=x_{1} i(S) x_{2}$ reduced, with $a(x)=$ $\# S$. Similarly, we can write $y=y_{1} i(T) y_{2}$ reduced, with $a(y)=\# T$. We can further assume that $i(S) x_{2}$ and $y_{1} i(T)$ satisfy the conditions of lemma 5.5.4 with 
$x^{\prime}=i(S) x_{2}$ and $y^{\prime}=y_{1} i(T)$. To see this, note that if there exists $t \in \mathcal{L}\left(i(S) x_{2}\right)$ which can be left cancelled, then there exists $s \in \mathcal{L}\left(x_{2}\right)$ which commutes with all elements of $S$ except $t$. Then we can write $t i\left(S^{\prime}\right) x_{2}^{\prime}$ where $S^{\prime}=(S \backslash\{t\}) \cup\{s\}$ and $x_{2}^{\prime}=s x_{2}$. We can continue such "shifting" until the conditions of lemma 5.5.4 are appropriately satisfied.

By proposition 4.4.2 and its dual, there exist $x^{\prime}$ and $y^{\prime}$ such that $b_{x^{\prime}} b_{x}=b_{i(S) x_{2}}$ and $b_{y} b_{y^{\prime}}=b_{y_{1} i(T)}$. We compute that $b_{x^{\prime}} b_{x} b_{y} b_{y^{\prime}}=b_{i(S) x_{2}} b_{y_{1} i(T)}=q_{2}{ }^{m} b_{w^{\prime}}$. By positivity, we conclude that $b_{x} b_{y}=q_{2}{ }^{m} b_{v}$ for some $v \in W_{c}$ and that $m \leq$ $\min (a(x), a(y))$, by lemma 5.5.3.

For equality to occur in the conclusion of the theorem, we must have $a(x)=a(y)$, so we assume this as well. By lemma 5.5.4, we have $m=\min (a(x), a(y))$ if and only if $i(S) x_{2}=i(T) y_{1}^{-1}$.

To finish the proof of theorem 5.5.1, it suffices to show that $v^{2}=1$ if and only if $x=y^{-1}$. By lemma 5.2.5, we know that $b_{i(S) x_{2}} b_{y_{1} i(T)}=q_{2} \# S b_{i(S)}$. By proposition 4.4.4, there exists a unique involution $d$ such that $d \sim_{R} x_{1} i(S)$. We know that the map $F: R(i(S)) \rightarrow R(d)$ defined by ${q_{2}}^{m^{\prime}} b_{F(i(S) u)}=b_{x_{1}} b_{i(S) u}$ is a bijection, since $b_{x^{\prime}} b_{x_{1} i(S)}=b_{i(S)}$. Therefore, there is only one element $y_{2}$ for which $v^{2}=1$ and by lemma 3.2.1, it is $y_{2}=x_{1}^{-1}$. Thus, $x=y^{-1}$ and the theorem follows.

\subsection{Proof of semi-simplicity.}

Theorem 5.6.1. The algebra $\overline{\mathcal{H}}$ is semi-simple.

Adjoin $\sqrt{q_{2}}$ to our ground field and rescale our basis so that $b_{w}^{\prime}=\frac{1}{q_{2^{a}}^{a(w) / 2}} b_{w}$. We then see that $\left(b_{x}^{\prime}, b_{y}^{\prime}\right)=\left(b_{x}, b_{y}\right) / q_{2}(a(x)+a(y)) / 2$. By theorem 5.5.1, we see that $\left(b_{x}^{\prime}, b_{y}^{\prime}\right)$ is 1 if $x=y$ and is either 0 or a negative power of $q_{2}$ for $x \neq y$.

In any case, in the limit as $q_{2} \rightarrow \infty$, the basis $\left\{b_{w}^{\prime}\right\}$ becomes an orthonormal basis. We conclude that there exist values of $q_{2}$ for which the form is positive definite as a form over the reals. Semi-simplicity follows.

\section{IRREDUCIBLE REPRESENTATIONS AND THEIR DIMENSIONS}

6.1. Representations. We shall now describe the irreducible representations of $\overline{\mathcal{H}}$.

By definition, the span of the elements $\left\{b_{w^{\prime}} \mid w^{\prime} \leq_{L} w\right\}$ constitutes a left ideal $I_{w}^{\prime}$ in $\overline{\mathcal{H}}$. Define $I_{w}=I_{w}^{\prime} /\left\langle b_{w^{\prime}} \mid w^{\prime}<_{L} w\right\rangle . I_{w}$ is a representation of $\overline{\mathcal{H}}$ with dimension $\# L(w)$. Depending on the context, we shall denote the canonical projection of $b_{v}$ in $I_{w}$ also by $b_{v}$.

Lemma 6.1.1. If $w \sim_{L R} w^{\prime}$ then $I_{w} \cong I_{w^{\prime}}$.

Proof. We define a map $f: I_{w} \rightarrow I_{w^{\prime}}$ as follows. By the dual lemma to lemma 4.2.5, there exist $x \in W_{c}$ and $S \in P$ such that every $v \in L(w)$ can be written $v=v^{\prime} i(S) x$ reduced, where $a(w)=\# S$. We also know that $w \sim_{L} i(S) x$. Let $x^{\prime}$ be such that $b_{i(S)}=b_{i(S) x} b_{x^{\prime}}$. Since $w \sim_{L R} w^{\prime}$ we know that $w^{\prime} \sim_{L R} i(S)$. Thus $b_{w^{\prime}}=b_{a} b_{i(S)} b_{b}$ for some $a, b \in W_{c}$. Let $b_{z}=b_{i(S)} b_{b}$. Then $w^{\prime} \sim_{L} z$. Thus, $I_{w^{\prime}}=I_{z}$. By lemma 4.2.3, there exists $z^{\prime}$ such that $z=i(S) z^{\prime}$ reduced. By lemma 4.4.3, there exists $z^{\prime \prime}$ such that $b_{z} b_{z^{\prime \prime}}=b_{i(S)}$. Let $f\left(b_{v}\right)=b_{v} b_{x^{\prime}} b_{z^{\prime}}$.

Since $v=v^{\prime} i(S) x$ as above, we see that $b_{v} b_{x^{\prime}} b_{z^{\prime}}=b_{v^{\prime}} b_{i(S) z^{\prime}}$. Since $a\left(v^{\prime} i(S)\right)=$ $\# S$, we see that $f\left(b_{v}\right) \in I_{w^{\prime}}^{\prime}$ as desired. Note that $b_{v}=f\left(b_{v}\right) b_{z^{\prime \prime}} b_{x}$, so that $f$ is an isomorphism. 
Since $f$ is defined using right multiplication, it is a left module isomorphism. The lemma follows.

Theorem 6.1.2. The irreducible representations are parametrized by $\bar{P}$. The bijection is given by $\bar{S} \mapsto I_{i(S)}$ where $\bar{S}$ is the equivalence class of $S \in P$.

Proof. Consider a two-sided cell $T(w)$. By theorem 4.5.1, there exists an $S \in P$ such that $i(S) \in T(w)$. By proposition 4.4.3 and its dual, $L(i(S))=\left\{v^{-1} \mid v \in R(i(S))\right\}$. Since every $w^{\prime} \in T(w)$ satisfies $b_{w}^{\prime}=b_{a} b_{i(S)} b_{b}$ for some $a$ and $b \in W_{c}$, we know that every $w^{\prime} \in T(w)$ is in some $L(v)$ for $v \in R(i(S))$. Thus, the number of left cells is bounded by \#R(i(S)).

We claim that the number of left cells in $T(w)$ is equal to \#R(i(S)). We shall prove this by induction on $\# S$, the case where $\# S=0$ being clear.

If there are fewer left cells than \#R(i(S)) in $T(i(S))$, then there must be a left cell, say $L(i(S) v)$ which intersects $R(i(S))$ in more than 1 element. Let $x$ be such that $b_{i(S) v} b_{x}=b_{i(S)}$. The map $F: L(i(S) v) \rightarrow L(i(S))$ defined by $b_{F z}=b_{z} b_{v}$ is a bijection since $b_{i(S) v} b_{z} b_{v}=b_{i(S) v}$. Therefore, we can assume that $L(i(S))$ intersects $R(i(S))$ in more than 1 element.

Suppose $i(S), i(S) y \in L(i(S)) \cap R(i(S))$. There exists $y^{\prime}$ such that $b_{y^{\prime}} b_{i(S)}=$ $b_{i(S) y}$. By lemma 4.2.3, we can assume that $y^{\prime} i(S)$ is reduced. Thus $y^{\prime} i(S)=i(S) y$.

Suppose $s \in S$ commutes with $\operatorname{supp}(y)$. Then $a(\operatorname{si}(S) y)=\# S-1$ and we would have $y^{\prime} i(S \backslash\{s\})=i(S \backslash\{s\}) y$. This is impossible, by our induction hypothesis.

Thus, we can assume no $s \in S$ commutes with $\operatorname{supp}(y)$. It follows that $y=v^{\prime} i(S)$ and $y^{\prime}=i(S) v$. We conclude that $v=v^{\prime}$ so that $i(S) \sim_{L} i(S) v i(S)$, where $i(S) v i(S)$ is reduced.

To finish the argument, we must appeal to the particular nature of $\Gamma$. The argument in type $A$ and $D$ is simpler than the argument for type $E$, which we now give. Since we made no particular choice of $S$, except that $i(S) \in T(w)$, we choose $S$ so that it involves the generator furthest from the branch point, which we call $N$ (see lemma 8.1.2). We then see that $i(S) v i(S)$ involves two occurrences of $N$. By lemma 8.1.6, we deduce that $i(S)=N$, so that $a(w)=1$. But by lemma 8.1.6, we see that we cannot have $i(S)=N$ and $a(w)=1$ since any expression with two occurrences of $N$ must also contain a substring consisting of the product of two commuting generators.

We conclude that the number of left cells in $T(i(S))$ is equal to \#R(i(S)). By lemma 6.1.1, any two such left cells are isomorphic. By semi-simplicity, we conclude that $I_{w}$ is simple and that the various two-sided cells carry non-isomorphic modules. The theorem follows.

Note that, in addition, we have $\# T(w)=\# R(i(S))^{2}$.

Corollary 6.1.3. We have $\# W_{c}=\sum_{\bar{S} \in \bar{P}} \operatorname{dim} I_{i(S)}^{2}=\sum_{\bar{S} \in \bar{P}} \# L(i(S))^{2}$.

Because of proposition 4.4.4, we also have the following.

Corollary 6.1.4. The number of involutions in $W_{c}$ is equal to $\sum_{\bar{S} \in \bar{P}} \operatorname{dim} I_{i(S)}=$ $\sum_{\bar{S} \in \bar{P}} \# L(i(S))$.

Corollary 6.1.3 was used when $W$ itself is finite in order to compute the cardinalities of $W_{c}$ in [2] and [3]. Nevertheless, it is amusing to observe the dimensions in type $A$ and $D$ from the perspective of cells. We describe these next without proof. (Proofs are similar and simpler than the argument we give for type E.) 
6.2. Dimensions in type $A$ and $D$. In the following tables, the column headed "Rank" shows $\# \Gamma_{g}$, the column headed "\# $W_{c}$ " shows $\# W_{c}$, and the remaining columns are headed by the value of the $a$-function on an element in the two-sided cell which carries the corresponding irreducible representation.

We denote the Catalan numbers by $C_{n}=\frac{1}{n+1}\left(\begin{array}{c}2 n \\ n\end{array}\right)$.

We have the following table in type $A$.

$\begin{array}{rrrrrrr}\text { Rank } & \# W_{c} & 0 & 1 & 2 & 3 & \\ 1 & 2 & 1 & 1 & & & \\ 2 & 5 & 1 & 2 & & & \\ 3 & 14 & 1 & 3 & 2 & & \\ 4 & 42 & 1 & 4 & 5 & & \\ 5 & 132 & 1 & 5 & 9 & 5 & \\ 6 & 429 & 1 & 6 & 14 & 14 & \\ 7 & 1430 & 1 & 7 & 20 & 28 & 14 \\ 8 & 4862 & 1 & 8 & 27 & 48 & 42\end{array}$

This table is like Pascal's triangle in that each number is the sum of the appropriate two numbers above it with the difference being that there are two rows each with the same number of elements. The irreducible representations correspond to Young diagrams with two or fewer columns (where we take the single column diagram to represent the sign representation) and have dimensions $\left(\begin{array}{c}n+1 \\ a\end{array}\right)-\left(\begin{array}{c}n+1 \\ a-1\end{array}\right)$, where $a$ is the value of the $a$-function. We have $\# W_{c}=C_{n+1}$.

In type $D$ we have the following table.

$\begin{array}{rrrrrrrr}\text { Rank } & \# W_{c} & 0 & 1 & 2 & 3 & 4 & 5 \\ 2 & 4 & 1 & 1,1 & 1 & & & \\ 3 & 14 & 1 & 3 & 2 & & & \\ 4 & 48 & 1 & 4 & 3,3,3 & 2 & & \\ 5 & 167 & 1 & 5 & 10,4 & 5 & & \\ 6 & 593 & 1 & 6 & 15,5 & 10,10,9 & 5 & \\ 7 & 2144 & 1 & 7 & 21,6 & 35,14 & 14 & \\ 8 & 7864 & 1 & 8 & 28,7 & 56,20 & 35,35,28 & 14 \\ 9 & 29171 & 1 & 9 & 36,8 & 84,27 & 126,48 & 42\end{array}$

We have taken $D_{2}=A_{1} \times A_{1}$ and $D_{3}=A_{3}$. The dimensions $>1$ for type $A_{n}$ appear as the last numbers in each column with $a$ value $>1$ in the row for $D_{n+1}$. This is because representations of $A_{n}$ lift to representations of $D_{n+1}$ for which the action of the two commuting end generators are the same. The other numbers constitute half of Pascal's triangle, with the exception that when the rank is $2 m$, there are two representations of $a$-value $m$ with dimension $\frac{1}{2}\left(\begin{array}{c}2 m \\ m\end{array}\right)$. We have $\# W_{c}=\frac{n+3}{2} C_{n}-1$ (see [2], [3], or [15]).

6.3. Notation for type $E$. In the remainder of this section, we shall work in rank $N+1$ and label the generators $\Gamma_{g}$ by the numbers 0 through $N$ in such a way that 1 through $N$ generate a subgroup of type $A_{N}$ with $I$ commuting with $J$ unless $|I-J|=1$ for $I, J>0$, and so that 3 corresponds to the branch node. The remaining node (which is joined to 3 ) is labelled 0.

By "consecutive occurrences" of a generator in a reduced expression, we mean two occurrences of that generator with no appearance of the same generator in between. We shall sometimes write $\overline{\mathcal{H}}_{n}$ for $\overline{\mathcal{H}}$ in case $\Gamma$ is of type $E_{n}$. Note that $\overline{\mathcal{H}}_{n}$ is naturally a subalgebra of $\overline{\mathcal{H}}_{n+1}$. 
Let $P^{\prime}$ be the subset of $P$ consisting of those $S$ such that the following three conditions hold: (1) If $S \neq \emptyset$, then $N \in S$, (2) if $K \in S, K>0$, then $K+2 \in S$, and (3) if $0 \in S$, then $4 \in S$. By lemma 8.1.2, $P^{\prime}$ constitutes a set of equivalence class representatives for $\sim$ in $P$.

Explicitly, when $N$ is even, $P^{\prime}$ consists of the sets $\{N, N-2, N-4, \ldots, N-2 K\}$, $0 \leq K \leq N / 2$, the set $\{N, N-2, N-4, \ldots, 4\} \cup\{0\}$, and the empty set. When $N$ is odd, $P^{\prime}$ consists of the sets $\{N, N-2, N-4, \ldots, N-2 K\}, 0 \leq K \leq N / 2$, and the empty set.

Let $L(i(S))_{2}$ denote the set of $w \in L(i(S))$ such that $N$ occurs twice in a reduced expression for $w$. (Note that the number of occurrences of a generator in a reduced expression for $w \in W_{c}$ does not depend on the reduced expression.) Similarly, define $R(i(S))_{2}$.

Define three sequences $f_{k}^{0}, f_{k}^{1}$, and $f_{k}^{2}$ by the following linear recurrence relations:

$$
\begin{aligned}
f_{k+1}^{0} & =f_{k}^{1}+f_{k}^{2}, \\
f_{k+1}^{1} & =f_{k}^{2}+f_{k-1}^{2}, \\
f_{k+1}^{2} & =f_{k}^{0}
\end{aligned}
$$

for $k>1$ and setting $f_{0}^{0}=f_{1}^{0}=0, f_{0}^{1}=-1, f_{1}^{1}=0, f_{0}^{2}=1$, and $f_{1}^{2}=0$.

Also let $f_{k}=f_{k}^{0}+f_{k}^{1}+f_{k}^{2}$.

The first few terms in these sequences go like this:

$$
\begin{array}{rrrrrrrrrrr}
f_{k}^{0} & 0 & 0 & 0 & 1 & 0 & 1 & 1 & 2 & 2 & 4 \\
f_{k}^{1} & -1 & 0 & 1 & 0 & 0 & 1 & 1 & 1 & 2 & 3 \\
f_{k}^{2} & 1 & 0 & 0 & 0 & 1 & 0 & 1 & 1 & 2 & 2 \\
f_{k} & 0 & 0 & 1 & 1 & 1 & 2 & 3 & 4 & 6 & 9
\end{array}
$$

We remark that $\sum_{k=0}^{\infty} f_{k}^{0} x^{k}=\frac{x^{3}}{1-x^{2}-x^{3}-x^{4}}$.

6.4. Dimensions in type $E$. We have the following table in type $E$.

$\begin{array}{rrrrrrrrrr}\text { Rank } & \# W_{c} & 0 & 1 & 2 & 3 & 4 & 5 & 6 & 7 \\ 3 & 10 & 1 & 2,1 & 2 & & & & & \\ 4 & 42 & 1 & 4 & 5 & & & & & \\ 5 & 167 & 1 & 5 & 10,4 & 5 & & & & \\ 6 & 662 & 1 & 6 & 20 & 15 & & & & \\ 7 & 2670 & 1 & 7 & 27 & 35,21 & 15 & & & \\ 8 & 10846 & 1 & 8 & 35 & 84 & 50 & & & \\ 9 & 44199 & 1 & 9 & 44 & 120 & 135,84 & 50 & & \\ 10 & 180438 & 1 & 10 & 54 & 165 & 340 & 186 & & \\ 11 & 737762 & 1 & 11 & 65 & 220 & 506 & 527,341 & 187 & \\ 12 & 3021000 & 1 & 12 & 77 & 286 & 727 & 1376 & 715 & \\ 13 & 12387990 & 1 & 13 & 90 & 364 & 1014 & 2105 & 2093,1377 & 715 \\ 14 & 50864885 & 1 & 14 & 104 & 455 & 1379 & 3121 & 5578 & 2809\end{array}$

We have taken $E_{3}=A_{2} \times A_{1}, E_{4}=A_{4}$, and $E_{5}=D_{5}$. Note that in odd ranks, there are two irreducible representations with the penultimate $a$-function value.

Let $d_{k}^{n}$ be the dimension of the irreducible representation of $\overline{\mathcal{H}}_{n}$ with $\# \Gamma_{g}=n$ and $a$-value $k$, except when $n=2 m+1$ and $k=m$. In this case, let $d_{m}^{2 m+1,1}$ be the dimension of the irreducible representation $I_{i(S)}$ where $S \in P$ has $\# S=m$ and its equivalence class under $\sim$ consists of exactly one element. Denote by $d_{m}^{2 m+1}$ the dimension of the other irreducible representation with $a$ value $m$ (and in the same 
row). In our table, $d_{m}^{2 m+1}$ is listed before $d_{m}^{2 m+1,1}$. For convenience, define $d_{-1}^{n}=0$ for all $n$.

Proposition 6.4.1. We have the following recurrence relations valid for $m>1$ :

$$
\begin{aligned}
d_{k}^{2 m} & =d_{k}^{2 m-1}+d_{k-1}^{2 m-1}+f_{k} \text { for } k<m-1, \\
d_{m-1}^{2 m} & =d_{m-1}^{2 m-1,1}+d_{m-1}^{2 m-1}+d_{m-2}^{2 m-1}+f_{k}, \\
d_{m}^{2 m} & =d_{m}^{2 m-1}+d_{m-1}^{2 m-1}+f_{m}^{1}, \\
d_{k}^{2 m+1} & =d_{k}^{2 m}+d_{k-1}^{2 m}+f_{k} \text { for } k<m, \\
d_{m}^{2 m+1} & =d_{m}^{2 m}+d_{m-1}^{2 m}+f_{m}^{1}+f_{m}^{2}, \\
d_{m}^{2 m+1,1} & =d_{m-1}^{2 m}+f_{m}^{0}, \\
d_{m+1}^{2 m+1} & =d_{m}^{2 m}+f_{m-1}^{2} .
\end{aligned}
$$

We have $d_{0}^{3}=1, d_{1}^{3}=2, d_{1}^{3,1}=1$, and $d_{2}^{3}=1$.

This table can be interpreted in terms of restriction of representations if we replace $d$ by the corresponding representation $I_{w}$ and interpret $f_{k}^{i}$ as $f_{k}^{i}$ multiples of the trivial representation.

Lemma 6.4.2. Let $S \in P^{\prime}$. Note that $L(i(S))$ parametrizes a basis of $I_{i(S)}$. Let $I_{i(S)}^{1}$ be the span of $\left\{b_{w} \mid w \in L(i(S)) \backslash L(i(S))_{2}\right\}$ in $I_{i(S)}$. Then $I_{i(S)}^{1}$ is stable under the action of $\overline{\mathcal{H}}_{N}$. Furthermore, $I_{i(S)} / I_{i(S)}^{1}$, as an $\overline{\mathcal{H}}_{N}$-module, is a direct sum of $\# L(i(S))_{2}$ one dimensional representations.

Proof. Let $w \in W_{c}$. Define $w^{\prime}$ by $c b_{w^{\prime}}=b_{K} b_{w}$. If $K<N$ then the number of occurrences of $N$ in $w^{\prime}$ cannot exceed the number of occurrences of $N$ in $w$. Therefore, $I_{i(S)}^{1}$ is $\overline{\mathcal{H}}_{N}$-stable.

Let $w \in L(i(S))_{2}$ and write $w=g^{-1} 20 g x$ as in the dual to corollary 8.1.6. Observe that

$$
\begin{aligned}
b_{K} b_{g^{-1} 20 g} & =b_{N(N-1) \cdots(K+2)} b_{K(K-1) \cdots 3} b_{20 g} \\
& =b_{K(K-1) \cdots 3} b_{20} b_{345 \cdots K} b_{N(N-1) \cdots(K+2)} b_{(K+1) \cdots N} \\
& =b_{K(K-1) \cdots 3} b_{20} b_{345 \cdots K} b_{N} .
\end{aligned}
$$

Thus, $b_{K} b_{w}=b_{w^{\prime}}$ (by proposition 5.4.1) where $w^{\prime} \notin L(i(S))_{2}$ provided that $K<N$. We conclude that the one dimensional subspace spanned by the projection of $b_{w}$ in $I_{i(S)} / I_{i(S)}^{1}$ is $\overline{\mathcal{H}}_{N}$ stable. Lemma 6.4.2 follows.

We now prove proposition 6.4.1. Lemma 8.1.7 gives the dimension of $I_{i(S)} / I_{i(S)}^{1}$. Thus, it suffices to check that the representation $I_{i(S)}^{1}$ is an appropriate sum of irreducible representations when restricted to $\overline{\mathcal{H}}_{N}$.

Let $J^{-}=\left\{w \in L(i(S)) \backslash L(i(S))_{2} \mid w N \sim_{L R} i(S \backslash\{N\})\right\}$.

Let $J^{+}=\left\{w \in L(i(S)) \backslash L(i(S))_{2} \mid w N \sim_{L R} i(S)\right\}$.

Let $I^{+}$be the span of the elements $\left\{b_{w} \mid w \in J^{+}\right\}$. By 4.2.2, we know that $I^{+}$is a stable subspace in $I_{i(S)}$. Let $I^{-}=I_{i(S)}^{1} /\left(I_{i(S)}^{1} \cap I^{+}\right)$. Note that $J^{-}$parametrizes a basis for $I^{-}$.

The map induced by the map $w \mapsto w N$ restricted to $J^{-}$induces an isomorphism between $I^{-}$and the representation $I_{i(S \backslash\{N\})}$ for $\overline{\mathcal{H}}_{N}$. The inverse map is given by the map induced by $w \mapsto w N$.

We now consider cases. 
Case 1. Suppose $N$ is even and $\# S=1+N / 2$. Then $J^{+}$is empty since the maximum value of the $a$-function for rank $N$ is $N / 2$.

Case 2. Suppose $N$ is even, $\# S=N / 2$, and $0 \in S$. Here, the equivalence class of $S$ consists of a unique element. Let $w \in L(i(S))$. If $w N \sim_{L R} i(S)$, then there must be a reduced expression of $w N$ which involves a substring of the form $i(S)$, since the only $T \in P$ equivalent to $S$ is $S$. However $N \in S$, but $N \notin \operatorname{supp}(w N)$. Therefore $J^{+}$is empty.

Case 3. Suppose $\# S<\frac{N-2}{2}$. Let $I^{+}$be the span of $\left\{b_{w} \mid w \in J^{+}\right\}$. Let $T=$ $\{K-1 \mid K \in S\}$. Let $w \in J^{+}$.

By lemma 8.1.8, $\mathcal{R}(w i(S))=T$.

Thus, we can define a map $p: J^{+} \rightarrow L(i(T))$ by $p(w)=w^{\prime}$ such that $b_{w^{\prime}}=$ $b_{w N} b_{i(T)}$. This map induces an $\overline{\mathcal{H}}_{N}$-module homomorphism $p^{\prime}$ from $J^{+}$to $I_{i(T)}$ which is a non-zero. Similarly, the map from $I_{i(T)}$ (relative to $\overline{\mathcal{H}}_{N}$ ) into $I^{+}$which sends $b_{w}$ to $b_{w} b_{i(S)}$ is an $\overline{\mathcal{H}}_{N}$ module homomorphism $q$. Since $q$ and $p^{\prime}$ are inverse to each other, we see that $I^{+}$is isomorphic to the $\overline{\mathcal{H}}_{N}$ module $I_{i(T)}$.

Case 4. Suppose that $N$ is even and $\# S=N / 2$ but $0 \notin S$. We can apply the same reasoning as in case 3 , once we recall that, in the notation of the previous case, we cannot have $0 \in \mathcal{R}(w i(S))$ since $S$ is not equivalent to $T \cup\{0\} \backslash\{2\}$.

Case 5. Suppose that $N$ is odd and $\# S=(N-1) / 2$. Here we reason as in case 3 except that we note that $p$ maps onto $L(i(T)) \cup L\left(i\left(T^{\prime}\right)\right.$ ) (sets defined relative to $\left.\overline{\mathcal{H}}_{N}\right)$, where $T^{\prime}$ represents the unique equivalence class of cardinality $(N-1) / 2$ which contains one member. Also, the range of $p^{\prime}$ and domain of $q$ must be changed to $I_{i(T)} \oplus I_{i\left(T^{\prime}\right)}$.

Case 6. Suppose that $N$ is odd and $\# S=(N+1) / 2$. Here, the reasoning of case 3 goes straight through.

This exhausts the possibilities. Proposition 6.4.1 is proven.

6.5. Generating functions. Noam Elkies has worked out generating functions for the dimensions of the irreducible representations in type $E$, and the author has applied his techniques to $F$ and $H$. We hope to explain these in a sequel. For instance, in the notation of section 7.2 , let $C_{2, o}(y)=\sum_{k \geq 1} d_{k}^{2 k+1,2} y^{k-1}$.

Let $S(y)=\sqrt{1-4 y}$ and let $D(y)$ denote the product

$\left(y^{2}+y-1\right)(1+2 y+S(y))\left(1-5 y+2 y^{2}+(3 y-1) S(y)\right)^{2}\left(-1+3 y+2 y^{2}+(1-y) S(y)\right)$.

Then

$$
\begin{aligned}
C_{2, o}(y) & =\frac{-8}{D(y)}\left(6-77 y+352 y^{2}-650 y^{3}+293 y^{4}+338 y^{5}-249 y^{6}-3 y^{7}+2 y^{8}\right. \\
& \left.+\left(-6+65 y-234 y^{2}+288 y^{3}+15 y^{4}-186 y^{5}+41 y^{6}+5 y^{7}\right) S(y)\right) .
\end{aligned}
$$

Stembridge [13] has found generating functions for the total number of "fully commutative" elements (see section 7) using quite different methods.

\section{THE NON-SIMPLY LACED CASES}

In [7], Graham constructs algebras analogous to $\overline{\mathcal{H}}$ for non-simply laced cases as well. He shows that these algebras have a basis parametrized by the elements Stembridge has termed "fully commutative" [14]. 
Fully commutative elements of an arbitrary Coxeter group are those elements for which any reduced expression can be obtained from any other via a sequence of commutation relations.

Both Stembridge [14] and Graham [7] classified all Coxeter groups for which the number of fully commutative elements is finite. They obtained seven infinite families: $A_{n}, B_{n}, D_{n}, E_{n}, F_{n}, H_{n}, I_{2}(m)$. The Coxeter graph for the $F$ series is like that of type $A$ only there is a double bond placed between the second and third nodes. The Coxeter graph for the $H$ series is like that of $A$ except that the first two nodes, $s$ and $t$, satisfy $(s t)^{5}=1$.

The appropriate analogue of $\overline{\mathcal{H}}$ for the non-simply laced cases is to take the Hecke algebra $\mathcal{H}$ and quotient by the two sided ideal generated by the elements $\sum_{w \in W_{2}} T_{w}$ for each irreducible rank 2 proper parabolic subgroup $W_{2}$ of $W$.

Note that in types $A_{n}, B_{n}, D_{n}, E_{6}, E_{7}, E_{8}, F_{4}, H_{3}, H_{4}$, and $I_{2}(m)$, the Hecke algebra is already generically semisimple so that $\overline{\mathcal{H}}$ is also generically semisimple in these cases.

In type $I_{2}(m)$, there are either 2 two-sided cells or 4 two-sided cells, depending on whether $I_{2}(m)$ has 2 or 4 one dimensional irreducible representations. All the two dimensional representations of $I_{2}(m)$ are carried by the left cells in the biggest two-sided cell.

7.1. Type $B$ and $F$. When $\Gamma$ is of type $B$ or $F, \overline{\mathcal{H}}$ is the $\mathbb{Q}\left(q^{1 / 2}\right)$-algebra generated by $b_{s}, s \in S$, and satisfying all the same relations as in the simply-laced case with the addition that $b_{s} b_{t} b_{s} b_{t}=2 b_{s} b_{t}$ if stst $=t s t s$.

Because the monomial property still holds, many of our methods apply to these cases as well. Indeed, because there are no branch points in the graph, some proofs are simpler.

In particular, $\overline{\mathcal{H}}$ is semi-simple.

We now review the modifications that must be made to handle these cases. The following discussion is not meant to be complete. It is only meant to highlight the major differences.

First of all, $W_{c}$ must be replaced by the set of fully-commutative elements.

Property R3 is no longer true.

Properties R4, R4', R5, R5' must be changed as follows. Property R4 holds except that possibly, $w=w_{1} s w_{2} t w_{3} s w_{4}$ reduced, where $(s t)^{4}=1, t$ commutes with every $u \in \operatorname{supp}\left(w_{3}\right) \cup \operatorname{supp}\left(w_{4}\right)$, and $s$ commutes with every $u \in \operatorname{supp}\left(w_{2}\right) \cup$ $\operatorname{supp}\left(w_{3}\right)$. Property R5 holds except that possibly, $w=w_{1} s t s w_{2}$ reduced, where $(s t)^{4}=1, t$ commutes with every $u \in \operatorname{supp}\left(w_{2}\right)$, and if $w=w_{1}^{\prime} s^{\prime} t s^{\prime} w_{2}^{\prime}$ is another such expression, then $s=s^{\prime}$. Properties R $4^{\prime}$ and $\mathrm{R} 5^{\prime}$ are modified analogously.

The monomial basis now has structure constants which may have factors which are powers of 2 .

The notions of left and right cancellability must be appropriately modified to handle the new versions of properties R4 and R5.

The set $P$ no longer parametrizes elements $w$ for which no element of $\mathcal{L}(w)$ or $\mathcal{R}(w)$ can be left or right cancelled, respectively. Instead, let $Q$ be the set of $w \in W_{c}$ such that no element of $\mathcal{L}(w)$ can be left cancelled and no element of $\mathcal{R}(w)$ can be right cancelled. It can be shown that the support of an element of $Q$ consists of nodes no two of which are connected by a single bond.

The notion of $P$ can be replaced by the set of supports of elements in $Q$, although this new notion for $P$ relinquishes its importance to $Q$. Here, $\sim$ should be defined 
between elements of $Q$. The notion of neighbor must be replaced by declaring $w$, $w^{\prime} \in Q$ neighbors if and only if there exists $s \in \Gamma_{g}$ such that

$$
b_{w^{\prime}}=c b_{s} b_{w} b_{s}
$$

where $c$ is a rational integer. The equivalence relation which is the transitive closure of being neighbors is denoted $\sim$. The two-sided cells are parametrized by $Q / \sim$. Note that the map $Q \rightarrow P$ obtained by taking supports is not a bijection.

The $a$-function can be defined by

$$
b_{w^{-1}} b_{w}=c q_{2}^{a(w)} b_{d}
$$

where $c$ is a rational integer. This can be shown to be equivalent to the following. First define $a(w)$ as the number of connected components of $\operatorname{supp}(w)$ for $w \in Q$. Then define

$$
a(w)=\max \left\{a\left(w^{\prime}\right) \mid w^{\prime} \in Q, w=x w^{\prime} y \text { reduced }\right\} .
$$

Left cells and right cells are still indexed by involutions.

The matrix of the bilinear form with respect to the monomial basis will have entries of the form $2^{a} q_{2}{ }^{b}$.

7.2. Dimensions in type $B$ and $F$. In type $B$, it is convenient to split the representations into two kinds, those that can be lifted from the subalgebra of type $A_{n-1}$, and those that cannot. Any representation of $\overline{\mathcal{H}}$ of type $A_{n-1}$ can be lifted to a representation of $\overline{\mathcal{H}}$ of type $B_{n}$ by letting $b_{s}$ act as 0 where $s$ denotes the end node attached by a double bond to $\Gamma$.

We have the following table in type $B$ with the representations which come from type $A$ listed to the left of the column of ranks.

$\begin{array}{rrrrccrrrr}3 & 2 & 1 & 0 & \text { Rank } & \# W_{c} & 1 & 2 & 3 & 4 \\ & & 1 & 1 & 2 & 7 & 1,2 & & & \\ & & 2 & 1 & 3 & 24 & 1,3 & 3 & & \\ & 2 & 3 & 1 & 4 & 83 & 1,4 & 4,6 & & \\ & 5 & 4 & 1 & 5 & 293 & 1,5 & 5,10 & 10 & \\ 5 & 9 & 5 & 1 & 6 & 1055 & 1,6 & 6,15 & 15,20 & \\ 14 & 14 & 6 & 1 & 7 & 3860 & 1,7 & 7,21 & 21,35 & 35\end{array}$

We have $\# W_{c}=(n+2) C_{n}-1$, which was first shown by Stembridge [15]. The dimensions which do not come from type $A$ constitute Pascal's triangle without one of its borders.

In type $F$, let us label the generators 1 through $N$ where 2 and 3 are connected by the double bond. Let $Q_{0}$ consist of elements $w$ of the form

$$
N(N-2)(N-4) \cdots(N-2 k)
$$

such that $1,2 \notin \operatorname{supp}(w)$, including the identity element. Let $Q_{1}$ consist of elements $w \in Q$ of the form $1 w^{\prime}$ where $w^{\prime} \in Q_{0}$. Let $Q_{2}$ consist of elements $w \in Q$ of the form $23 w^{\prime}$ where $w^{\prime} \in Q_{0}$ and $\{2,3\} \subset \operatorname{supp}\left(23 w^{\prime}\right)$. Then $Q^{\prime}=Q_{0} \cup Q_{1} \cup Q_{2}$ constitute a complete set of equivalence class representatives for $\sim$ in $P$. 
We have the following table in type $F$, with the dimensions listed according to the families $Q_{k}$.

\begin{tabular}{rrrllllrllllrlrr} 
& \multicolumn{1}{c}{$Q_{0}$} & \multicolumn{1}{c}{$Q_{1}$} & \multicolumn{1}{c}{$Q_{2}$} & & \\
Rank & $\# W_{c}$ & 0 & 1 & 2 & 3 & 4 & 1 & 2 & 3 & 4 & 5 & 1 & 2 & 3 & 4 \\
3 & 24 & 1 & 1 & & & & 2 & 3 & & & & 3 & & & \\
4 & 106 & 1 & 2 & & & & 2 & 9 & & & & 4 & & & \\
5 & 464 & 1 & 3 & 2 & & & 2 & 12 & 9 & & & 5 & 14 & & \\
6 & 2003 & 1 & 4 & 5 & & & 2 & 15 & 36 & & & 6 & 20 & & \\
7 & 8560 & 1 & 5 & 9 & 5 & & 2 & 18 & 52 & 37 & & 7 & 27 & 57 & \\
8 & 36333 & 1 & 6 & 14 & 14 & & 2 & 21 & 71 & 148 & & 8 & 35 & 85 & \\
9 & 153584 & 1 & 7 & 20 & 28 & 14 & 2 & 24 & 93 & 221 & 149 & 9 & 44 & 121 & 235 \\
10 & 647775 & 1 & 8 & 27 & 48 & 42 & 2 & 27 & 118 & 316 & 608 & 10 & 54 & 166 & 358
\end{tabular}

The representations indexed by $Q_{0}$ are representations lifted from $A_{N-2}$. The elements $b_{3}, b_{4}, b_{5}, \ldots, b_{N}$ constitute a subalgebra of type $A_{N-2}$. A representation for this subalgebra can be extended to a representation of $\overline{\mathcal{H}}$ by letting $b_{1}$ and $b_{2}$ act as multiplication by zero.

Denote by $I_{k}^{n, l}$ the irreducible representation $I_{w}$ where $w$ is the unique element in $Q_{l}$ with $a$-value $k$. Denote by $d_{k}^{n, l}$ the dimension of $I_{k}^{n, l}$. Define $d_{-1}^{n, l}=0$ for convenience.

Let $\phi_{k}$ denote the Fibonacci sequence, where we take $\phi_{0}=1, \phi_{1}=0$, and $\phi_{k+2}=\phi_{k+1}+\phi_{k}$, for $k \geq 0$.

Proposition 7.2.1. We have the following recurrence relations valid for $n \geq 3$ and $m \geq 2$.

$$
\begin{aligned}
d_{k}^{n+1,0} & =d_{k}^{n, 0}+d_{k-1}^{n, 0} \text { for } k \leq(n+1) / 2, \\
d_{k}^{n+1,1} & =d_{k}^{n, 1}+d_{k-1}^{n, 1}+\phi_{k} \text { for } k<(n+1) / 2, \\
d_{m}^{2 m, 1} & =d_{m}^{2 m-1,1}+d_{m-1}^{2 m-1,1}+d_{m-1}^{2 m-1,2}+\phi_{m}, \\
d_{m+1}^{2 m+1,1} & =d_{m}^{2 m, 1}+\phi_{m-1}, \\
d_{1}^{n+1,2} & =d_{1}^{n, 2}+\phi_{2}, \\
d_{k}^{n+1,2} & =d_{k}^{n, 2}+d_{k-1}^{n, 2}+\phi_{k} \text { for } 1<k<(n+1) / 2, \\
d_{m}^{2 m+1,2} & =d_{m-1}^{2 m, 2}+d_{m}^{2 m, 1}+\phi_{m} .
\end{aligned}
$$

We have $d_{0}^{3,0}=d_{1}^{3,0}=1, d_{1}^{3,1}=2$, and $d_{2}^{3,1}=d_{1}^{3,2}=3$.

This table can be interpreted as restriction of representations if we replace $d$ by $I$ and interpret $\phi_{k}$ as $\phi_{k}$ multiples of the trivial representation.

7.3. Type $H$. When $\Gamma$ is of type $H, \overline{\mathcal{H}}$ is the $\mathbb{Q}\left(q^{1 / 2}\right)$-algebra generated by $b_{s}$ with the same relations as in the simply-laced case with the additional, non-monomial relation, $b_{s} b_{t} b_{s} b_{t} b_{s}=3 b_{s} b_{t} b_{s}-b_{s}$ when $(s t)^{5}=1$.

We are unsure to what extent our techniques apply to this case. Nevertheless, we have been able to deduce many facts which suggest that $\overline{\mathcal{H}}$ is semi-simple.

Conjecture 7.3.1. The algebra $\overline{\mathcal{H}}$ of type $H$ is semi-simple. 
In type $H$, the sizes of the left cells are as follows.

$\begin{array}{rrrrrrr}\text { Rank } & \# W_{c} & 0 & 1 & 2 & 3 & \\ 2 & 9 & 1 & 2+2 & & & \\ 3 & 44 & 1 & 3+3 & 5 & & \\ 4 & 195 & 1 & 4+4 & 9+9 & & \\ 5 & 804 & 1 & 5+5 & 14+14 & 19 & \\ 6 & 3185 & 1 & 6+6 & 20+20 & 34+34 & 69 \\ 7 & 12368 & 1 & 7+7 & 27+27 & 55+55 & \\ 8 & 47607 & 1 & 8+8 & 35+35 & 83+83 & 125+125\end{array}$

We have written $a+a$ instead of $2 a$ where we believe that the corresponding left cell carries a direct sum of two irreducible representations of the same dimension.

Let $l_{k}^{n}$ be the number of elements in any left cell consisting of elements with $a$-value $k$ for rank $n$, and let $I_{k}^{n}$ denote the corresponding $\overline{\mathcal{H}}$-module.

Proposition 7.3.2. We have the following formulas valid for $n \geq 2$.

$$
\begin{aligned}
& l_{0}^{n}=1, \\
& l_{k}^{n}=2\left(\left(\begin{array}{c}
n+1 \\
k
\end{array}\right)-1\right) \text { if } 0<k<(n+1) / 2, \\
& l_{k}^{n}=\left(\begin{array}{c}
n+1 \\
k
\end{array}\right)-1 \text { if } k=(n+1) / 2 .
\end{aligned}
$$

When $0<k \leq n / 2$, each left cell and each right cell in the two-sided cell with $a$-value $k$ intersect each other in exactly 2 elements. In other two-sided cells, each left cell intersects each right cell in a unique element.

We have $\# W_{c}=\left(\begin{array}{c}2 n+2 \\ n+1\end{array}\right)-2^{n+2}+n+3$.

Conjecture 7.3.3. If $k=0$ or if $n$ is odd and $k=\frac{n+1}{2}$, then $I_{k}^{n}$ is irreducible and of dimension $l_{k}^{n}$. Otherwise, $I_{k}^{n}$ is a direct sum of two irreducible representations each of dimension $l_{k}^{n} / 2$.

\section{APPENDiX}

This appendix contains facts about the combinatorics of reduced expressions in type $E$.

8.1. Combinatorics in type $E$. We shall use the notation explained in section 6.3 .

Lemma 8.1.1. Let $w \in W_{c}$. In any reduced expression for $w$, we claim that:

(1) Between any two occurrences of 0 , there must be at least two occurrences of 3 .

(2) Between any two occurrences of 3 , there must be generators from at least two of the three families $\{1,2\},\{0\}$, and $\{4, \ldots, N\}$.

(3) For any four consecutive occurrences of 3, at least one consecutive pair of these 3 's must be separated by generators other than 1 or 2 .

(4) Between any two occurrences of $N$, there must be an occurrence of two 3's separated by generators from the the set $\{0,1,2\}$ only.

Proof. For the proof, see [3, lemma 4].

Lemma 8.1.2. The set $P^{\prime}$ constitutes a complete set of equivalence class representatives for $P$ with respect to $\sim$. 
Proof. First note that the nodes $S \cap\{3,4,5, \ldots, N\}$ constitute a set equivalent to some set of the form $\{N, N-2, N-4, \ldots, N-2 k\}$. Note that $\{1,2\} \not \subset S$ since $12 \neq 21$. Thus $S \sim\{1\} \cup\{N, N-2, N-4, \ldots, N-2 k\} \cup S^{\prime}$, where $S^{\prime}$ is either $\{0\}$ or $\emptyset$. If $S^{\prime}=\emptyset$, we have $S \sim\{N, N-2, N-4, \ldots, N-2 k-2\}$. If $S^{\prime}=\{0\}$ and $N-2 k>4$, then $S \sim\{N, N-2, N-4, \ldots, N-2 k-2\} \cup\{1\}$, and this is equivalent to an element of $P^{\prime}$. If $S^{\prime}=\{0\}$ and $N-2 k=4$, then $S \sim\{N, N-2, N-4, \ldots, N-2 k-2\} \cup S^{\prime}$. In any case, we have shown that $S$ is equivalent to some member of $P^{\prime}$.

The fact that we get each equivalence class exactly once follows from the fact that almost all the sets in $P^{\prime}$ have different sizes, except when $N$ is even. In that case the result follows once we note that $\{N, N-2, N-4, \ldots, 4,0\}$ is not equivalent to any other $S \in P$.

Lemma 8.1.3. Let $w \in W_{c}$. Let $g=345 \cdots N$. If two occurrences of $N$ appear in a reduced expression for $w$, then we may write $w=x g^{-1} 02 y g z$ reduced. Furthermore we may assume that 02 appears just to the left of $g$, though this 02 may be the one which appears just to the right of $g^{-1}$.

We wish to point out explicitly that the statement of this lemma contains an ambiguity when we talk of 02 appearing "just to the left of $g$ ", for it may be that other substrings of the form $345 \cdots N$ appear elsewhere in the reduced expression. We hope it is clear that we mean the specific substring $345 \cdots N$ explicitly pointed out in the factorization of $w$. We feel that if we were to be completely precise in our wording, we would sacrifice clarity for precision. We hope our proofs, while technically containing these kinds of ambiguities, are nevertheless clear.

Proof. Consider two consecutive occurrences of $N$. By property R3, there must occur between them at least two occurrences of $N-1$. The first such occurrence must necessarily commute with every generator between it and the first of the two $N$ 's we are considering. Similarly, the last occurrence of $N-1$ in between the two $N$ 's commutes with all generators between it and the second of the two $N$ 's. Thus $w$ has a reduced expression of the form $\cdots N(N-1) \cdots(N-1) N \cdots$, where between the two identified $N-1$ 's there must occur, by property R3, at least two occurrences of $N-2$, etc. We continue until we have an expression as in the lemma.

The second assertion follows from part 4 of lemma 8.1.1 and the fact that 1 commutes with $\operatorname{supp}(g)$.

The following lemma also provides another proof of the finiteness of $W_{c}$ in type $E$ which we think is simpler than previous proofs.

Lemma 8.1.4. No element $w \in W_{c}$ has a reduced expression with four occurrences of $N$.

Finiteness of $W_{c}$ follows by induction on rank, since we know that $W_{c}$ in $E_{8}$ is a subset of a finite group.

Proof. Suppose $w$ violates the lemma. Write $w=x g^{-1} 02 y g z$ as in lemma 8.1.3 in such a way that the second and third occurrence of $N$ appear in $g^{-1}$ and $g$.

Since the first $N$ occurs left of the $g^{-1}$, there must be at least two occurrences of $N-1$ between the first $N$ and $g^{-1}$. The last of these $N-1$ 's, by property R3, implies the existence of at least one $N-2$ between this last $N-1$ and the $g^{-1}$. The last of these $N-2$ 's implies the existence of at least one $N-3$ between this last 
$N-2$ and the $g^{-1}$. Continuing we find that there exists an occurrence of 3 before $g^{-1}$ with no occurrences of 4 in between. This situation necessitates an occurrence of either a 0 or a 2 between the 3 and the $g^{-1}$, but because of the 0 just to the right of $g^{-1}$, there cannot be a 0 between the 3 and the $g^{-1}$. On the other hand an occurrence of 2 necessitates an occurrence of 1 just to the right of $g^{-1}$.

A similar argument shows that a 1 must appear just right of $g$. This is a contradiction if $y=$ identity. But if $y \neq i$ identity then the 02 just left of $g$ cannot equal the 02 just right of $g^{-1}$ and therefore, $3 \in \operatorname{supp}(y)$. Now a reduced expression for $y$ cannot contain a single 3 by part 1 of lemma 8.1.1. Consider the first 3. To its right there cannot be a 0 by part 1 of lemma 8.1.1. On the other hand, there cannot be a 2 , because that would necessitate a 1 , which together with the 1 just left of $g^{-1}$ would contradict $w \in W_{c}$. By part 2 of lemma 8.1.1, this is impossible, and our lemma follows.

Lemma 8.1.5. Let $S \in P$ be such that $N \in S$. Then no reduced expression for $w \in R(i(S))$ has three occurrences of $N$.

Proof. The proof is similar to the proof of lemma 8.1.4.

In this case, assume $w \in R(i(S))$ has a reduced expression involving three occurrences of $N$. Choose the reduced expression so that the first two $N$ 's are in the $g^{-1}$ and $g$ of lemma 8.1.3. Since a third $N$ occurs to the right of $g$, by the argument used to prove lemma 8.1.4, there must be a 1 just to the right of $g$. If $S=\emptyset$, there is nothing to prove, and if $S=\{N\}$, then we already have a contradiction since by lemma 8.1.3, there must be a reduced word with an occurrence of 02 , which violates $a(w)=\# S=1$. Otherwise, no $N-1$ can occur left of the first $N$ since $N \in \mathcal{L}(w)$. Since $\# S>1$, there must be some generator to the left of the first $N$ since nothing commutes with all of $\operatorname{supp}(g 02)$. By the argument in the proof of lemma 8.1.4, there must be a 1 just left of $g^{-1}$. We arrive at the same kind of contradiction as in the proof of lemma 8.1.4.

Examining these proofs, we have the following corollary.

Corollary 8.1.6. Let $S \in P$ contain $N$. Let $w \in R(i(S))_{2}$. Then we can write $w=x g^{-1} 02 g$ reduced.

Proposition 8.1.7. Let $S \in P^{\prime}$. Let $k=\# S$. We have

$$
\# R(i(S))_{2}=\left\{\begin{aligned}
f_{k} & \text { if } k<N / 2, \\
f_{k}^{1} & \text { if } k=(N+1) / 2 \text { and } N \text { odd }, \\
f_{k}^{1}+f_{k}^{2} & \text { if } k=N / 2, N \text { even, and } \# \bar{S}>1, \\
f_{k}^{0} & \text { if } k=N / 2, N \text { even, and } \# \bar{S}=1, \\
f_{k-2}^{2} & \text { if } k=N / 2+1 \text { and } N \text { even. }
\end{aligned}\right.
$$

Proof. We try to find a canonical reduced expression for any $w \in R(i(S))_{2}$. Temporarily assume $\# S<N / 2$.

Using lemma 8.1.6, write $w=x g^{-1} 02 g$ reduced. Recall that $\mathcal{L}(w)=S$. Therefore no $N-1$ occurs in $\operatorname{supp}(x)$. However, $N-2 \in S$ so that $N-2 \in \operatorname{supp}(x)$. Therefore, we can argue as in the proof of lemma 8.1.4 and see that $x=x^{\prime}(N-2) \cdots 321$ reduced. Also, $N-4 \in S$, so a similar argument shows that $x^{\prime}=x^{\prime \prime}(N-4) \ldots 30$. The tail of $x^{\prime}$ differs from that of $x$ in order not to violate the conclusions of 
lemma 8.1.1. We continue arguing in this manner until we have

$$
\begin{gathered}
x=y((N-2 k+2) \ldots 3) z_{k}((N-2 k+4) \ldots 3) z_{k-1} \\
\cdots((N-4) \cdots 3) 0((N-2) \cdots 321)
\end{gathered}
$$

reduced where the $z_{l}$ are one of the following words: 21,2 , or 0 . We also assume $N-2 k \notin S$.

We claim that $y=i d e n t i t y$. If not, then no element of $\mathcal{R}(y)$ may commute with the elements of $S$, otherwise $a(w)=\# S$ would be violated. However if $N-2 l-1 \in \mathcal{R}(y)$, then we must have an occurrence of $N-2 l$ to the right of $N-2 l-1$, which implies an occurrence of $(N-2 l-1)(N-2 l-2)$ to the right of $N-2 l-1$ with only one generator between the two mentioned $N-2 l-1$ 's which do not commute with $N-2 l-1$, namely the already mentioned $N-2 l-2$. This is a contradiction, so $y=i d e n t i t y$.

Thus, \#R(i(S)) depends on how many possibilities we have for the $z_{l}$.

Using lemma 8.1.1, we see that $z_{l}$ can be 2 if and only if $z_{l-1}=0$. Also, $z_{l}$ can be 21 if and only if $z_{l-1}$ is a 2 or, if $z_{l-1}=0$ and $z_{l-2}=2$. Finally, $z_{l}$ can be 0 if and only if $z_{l-1}=21$ or $z_{l-1}=2$. But these are just the linear recurrence relations defining the $f_{k}^{j}$. That is, $f_{k}^{0}$ is the number of $w$ such that $z_{k}=0, f_{k}^{1}$ is the number of $w$ such that $z_{k}=21$, and $f_{k}^{2}$ is the number of $w$ such that $z_{k}=2$. (Recall that here, $k$ has the special meaning that $N-2 k \notin S$ whereas $N-2 k+2 \in S$.)

We conclude that $\# R(i(S))=f_{k}$.

However, we made the assumption that $\# S<N / 2$. When $\# S \geq N / 2$, we proceed by cases.

Case 1. Suppose $\# S=(N+1) / 2$, so that $S=\{1,3,5, \ldots, N\}$. For this to happen, we must have $x=y(3) z_{k-1}(543) z_{k-2} \ldots((N-4) \cdots 3) 0((N-2) \cdots 321)$ reduced, where $y=1$. This is only possible if $z_{k}$ could be 21 , and for this, there are $f_{k}^{1}$ possibilities.

Case 2. Suppose $\# S=N / 2$ and $S=\{2,4,6, \ldots, N\}$. For this to happen, we must have $x=y(43) z_{k-1}(6543) z_{k-2} \ldots((N-4) \cdots 3) 0((N-2) \cdots 321)$ reduced, where $y=2$. This is only possible if $z_{k}$ could be 21 or 2 , and for this, there are $f_{k}^{1}+f_{k}^{2}$ possibilities.

Case 3. Suppose $\# S=N / 2$ and $S=\{0\} \cup\{4,6,8, \ldots, N\}$. For this to happen, we must have $x=y(43) z_{k-1}(6543) z_{k-2} \cdots((N-4) \cdots 3) 0((N-2) \cdots 321)$ reduced, where $y=0$. This is only possible if $z_{k}$ could be 0 , and for this, there are $f_{k}^{0}$ possibilities.

Case 4. Suppose $\# S=N / 2+1$. Then $S=\{0,2,4, \ldots, N\}$. For this to happen, we must have $x=y(43) z_{k-2}(6543) z_{k-3} \ldots((N-4) \cdots 3) 0((N-2) \cdots 321)$ reduced, where $y=021$. This is only possible if $z_{k-2}=2$, and for this, there are $f_{k-2}^{2}$ possibilities.

We have exhausted the possibilities. Lemma 8.1.7 follows.

We shall use the following lemma in the proof of lemma 6.4.2.

Lemma 8.1.8. Let $S \in P^{\prime}$ and $0 \notin S$. Let $w=x i(S)$ reduced with $a(w)=\# S$. Suppose that $w N \sim_{L R} i(S)$. Let $T=\{K-1 \mid K \in S\}$. We have $\mathcal{R}(w i(S))=T$, unless $N$ is odd and $\# S=(N-1) / 2$. If $N$ is odd and $\# S=(N-1) / 2$, then $\mathcal{R}(w i(S))$ is either $T$ or $T \cup\{0\} \backslash\{2\}$. 
Proof. Because $w N \sim_{L R} i(S)$, we can write $w N=x i(U) y$ reduced, where $U \sim S$. Choose such an expression which minimizes $l(y)$. Then, no element of $U$ is left cancellable in $i(U) y$, for if there were such an element, $y$ does not have minimal length.

Note that $a(i(U) y)=\# U=\# S$. Since $i(U) y N$ is a subword of $w$, we have, by lemma 4.2.2, $a(i(U) y N)=\# S$. Let $C$ be the set of generators in $S$ which commute with $\operatorname{supp}(i(U) y)$. Note that $N \notin C$, for otherwise, $a(i(U) y N)=\# U+1$. We must have $C \subset U$, since $a(w N)=\# U$. We can thus write $i(U) y=i\left(U^{\prime}\right) i(C) y^{\prime} i\left(R^{\prime}\right)$ reduced, where $U^{\prime}=U \backslash C$ and $R^{\prime}=\mathcal{R}(w N) \backslash C$.

We claim that $y^{\prime}=i d e n t i t y$.

To see this, first note that $a\left(i\left(U^{\prime}\right) y^{\prime} i\left(R^{\prime}\right)\right)=\# U^{\prime}=\# R^{\prime}+1$, and $i\left(U^{\prime}\right) y^{\prime} i\left(R^{\prime}\right)$ satisfies the conditions of proposition 4.3.4. Define $T_{k}$ and $\gamma_{k}$ as in lemma 4.3.2. By proposition 4.3.4, we know $a\left(y^{\prime} i\left(R^{\prime}\right)\right)=\# \mathcal{L}\left(y^{\prime} i\left(R^{\prime}\right)\right)$. Since $a\left(y^{\prime} i\left(R^{\prime}\right) N\right) \geq$ $\# R^{\prime}+1$, we must have $a\left(y^{\prime} i\left(R^{\prime}\right) N\right)=\# R^{\prime}+1$ and, therefore, $a\left(y^{\prime} i\left(R^{\prime}\right)\right)=\# R^{\prime}$ (by minimality of $l(y)$ ).

If $y^{\prime} \neq i$ dentity, then some element $s \in \mathcal{L}\left(y^{\prime}\right)$ is left cancellable in $y^{\prime} i\left(R^{\prime}\right)$. We also know that every element of $\mathcal{L}\left(y^{\prime}\right)$ is connected to at least two elements of $U^{\prime}$ since no element of $U^{\prime}$ is left cancellable. This implies that $s$ must be the branch generator, for otherwise, $i\left(U^{\prime}\right) y^{\prime} i\left(R^{\prime}\right) \notin W_{c}$. Furthermore, $s$ must be connected to precisely 2 elements of $U^{\prime}$. We conclude that every $s \in \mathcal{L}\left(y^{\prime}\right)$ is connected to exactly two elements of $U^{\prime}$, and every component of $\gamma_{1}$ is a graph of type $A$. This implies that any connected component of $\gamma_{1}$ must have one more node in $U^{\prime}$ than in $\mathcal{L}\left(y^{\prime} i\left(R^{\prime}\right)\right)$. Now, $\# \mathcal{L}\left(y^{\prime} i\left(R^{\prime}\right)\right)=\# R^{\prime}=\# U^{\prime}-1$. We conclude that $\gamma_{1}$ is connected.

Note that $N-1 \in U^{\prime}$, for $a\left(i\left(U^{\prime}\right) y^{\prime} i\left(R^{\prime}\right) N\right)=\# U^{\prime}$, and $\# R^{\prime}+1=\# U^{\prime}$, so that some element of $\mathcal{L}\left(i\left(U^{\prime}\right) y^{\prime} i\left(R^{\prime}\right) N\right)$ is left cancellable in $i\left(U^{\prime}\right) y^{\prime} i\left(R^{\prime}\right) N$, whereas no element of $\mathcal{L}\left(i\left(U^{\prime}\right) y^{\prime} i\left(R^{\prime}\right)\right)$ is left cancellable in $i\left(U^{\prime}\right) y^{\prime} i\left(R^{\prime}\right)$. This also shows that $N-1 \notin \operatorname{supp}\left(y^{\prime}\right)$. On the other hand, we know $C \cup U^{\prime}$ is a set of $\# S$ mutually commuting generators, which is impossible unless $C=\emptyset$. Thus $U=U^{\prime}$ and $R^{\prime}=\mathcal{R}(w N)=S \backslash\{N\}$. Furthermore, we must have $\mathcal{L}\left(y^{\prime}\right)=R^{\prime}$. Since $\mathcal{L}\left(y^{\prime}\right)$ contains the branch generator 3 , we have a contradiction when $N$ is even, and can conclude that $y^{\prime}=i d e n t i t y$. So assume $N$ is odd. Note that $3 \notin R^{\prime}$ unless $\# S \geq(N-1) / 2$. If $\# S=(N+1) / 2$, we must have $U=\{0,2,4,6, \ldots, N-1\}$, which contradicts the fact that $3 \in R^{\prime}$ is connected to exactly two nodes of $U$. If $\# S=(N-1) / 2$, then $R^{\prime}=\{3,5,7, \ldots, N-2\}$. If $y^{\prime} \neq i d e n t i t y$ in this case, then some $2 K \in \operatorname{supp}\left(y^{\prime}\right)$, where $K>1$, since otherwise, we could deduce from lemma 8.1.1 that $02 i(\{5,7,9, \ldots, N-2\})$ occurs as a subword in $y^{\prime} i\left(R^{\prime}\right)$ which would contradict $a\left(y^{\prime} i\left(R^{\prime}\right)\right)=(N-3) / 2$. But then we deduce from corollary 8.1.6 (applied to the case of rank $2 K+2$ ) that $y^{\prime}=i d e n t i t y$.

Thus, $i(U) y=i(U) i\left(R^{\prime}\right)$. The only way this is possible is if $U=\mathcal{R}(w i(S))$ as in the conclusion of the lemma. (Also note that every conclusion in the lemma is realizable.)

Lemma 8.1 .8 follows.

\section{REFERENCES}

1. N. Bourbaki, Groupes et algèbres de Lie, Chapitres 4, 5, et 6, Masson, Paris, 1981. MR 83g:17001

2. C. K. Fan, A Hecke Algebra Quotient and Properties of Commutative Elements of a Weyl Group, Thesis (1995), MIT, supervised by G. Lusztig. 
3. C. K. Fan, A Hecke Algebra Quotient and Some Combinatorial Applications, Journal of Algebraic Combinatorics 5 no 3 (1996), 175-189. CMP 96:14

4. C. K. Fan, Schubert Varieties and Short Braidedness, preprint (1996).

5. C. K. Fan and R. M. Green, Monomials and Temperley-Lieb Algebras, preprint (1996).

6. C. K. Fan and J. R. Stembridge, Nilpotent Orbits and Commutative Elements, preprint (1996).

7. J. J. Graham, Modular Representations of Hecke Algebras and Related Algebras, Thesis (1995), Univ. of Sydney.

8. V. F. R. Jones, Hecke algebra representations of braid groups and link polynomials, Annals of Mathematics, 126 (1987), 335-388. MR 89c:46092

9. - A polynomial invariant for knots via Von Neumann algebras, Bulletin of the Amer. Math. Soc., 12 no 1. (1985), 103-111. MR 86e:57006

10. L. H. Kauffmann and H. Saleur, An Algebraic Approach to the Planar Coloring Problem, Commun. Math. Phys. 152 (1993), 565-590. MR 94f:05056

11. D. Kazhdan and G. Lusztig, Representations of Coxeter groups and Hecke algebras, Invent. Math. 53 (1979), 165-184. MR 81j:20066

12. G. Lusztig, Cells in Affine Weyl Groups, in "Algebraic Groups and Related Topics," Advanced Studies in Pure Math. Vol. 6, Kinokuniya and North-Holland, Amsterdam, 1985. MR 87h:20074

13. J. R. Stembridge, The enumeration of fully commutative elements of Coxeter groups, preprint (1996).

14. J. R. Stembridge, On the Fully Commutative Elements of Coxeter Groups, Journal of Alg. Comb., to appear.

15. J. R. Stembridge, Some combinatorial aspects of reduced words in finite Coxeter groups, Trans. Amer. Math. Soc., to appear. CMP 96:12

16. H. N. V. Temperley and E. H. Lieb, Relations between the percolation and colouring problem ... , Proceedings of the Royal Society of London (1971), 251-280. MR 58:16425

17. B. W. Westbury, The representation theory of the Temperley-Lieb Algebras, Math. Z. 219 (1995), 539-565. MR 96h:20029

Abstract. Let $W$ be a Coxeter group with Coxeter graph $\Gamma$. Let $\mathcal{H}$ be the associated Hecke algebra. We define a certain ideal $\mathcal{I}$ in $\mathcal{H}$ and study the quotient algebra $\overline{\mathcal{H}}=\mathcal{H} / \mathcal{I}$. We show that when $\Gamma$ is one of the infinite series of graphs of type $E$, the quotient is semi-simple. We examine the cell structures of these algebras and construct their irreducible representations. We discuss the case where $\Gamma$ is of type $B, F$, or $H$.

Department of Mathematics, Harvard University, Cambridge, Massachusetts 02138

E-mail address: ckfan@math.harvard.edu

Current address: School of Mathematics, Institute for Advanced Study, Princeton, New Jersey 08540 